\title{
RIEMANN PROBLEM WITH DELTA INITIAL DATA FOR THE TWO-DIMENSIONAL STEADY PRESSURELESS ISENTROPIC RELATIVISTIC EULER EQUATIONS*
}

\author{
YU ZHANG ${ }^{1, * *}$ AND YANYAN ZHANG ${ }^{2}$
}

\begin{abstract}
The Riemann problem for the two-dimensional steady pressureless isentropic relativistic Euler equations with delta initial data is studied. First, the perturbed Riemann problem with three pieces constant initial data is solved. Then, via discussing the limits of solutions to the perturbed Riemann problem, the global solutions of Riemann problem with delta initial data are completely constructed under the stability theory of weak solutions. Interestingly, the delta contact discontinuity is found in the Riemann solutions of the two-dimensional steady pressureless isentropic relativistic Euler equations with delta initial data.
\end{abstract}

Mathematics Subject Classification. 35L65, 35L67, 76N10.

Received September 13, 2020. Accepted January 31, 2021.

\section{INTRODUCTION}

Consider the two-dimensional steady isentropic relativistic Euler equations

$$
\left\{\begin{array}{l}
\left(\frac{n u}{\sqrt{1-u^{2} / c^{2}-v^{2} / c^{2}}}\right)_{x}+\left(\frac{n v}{\sqrt{1-u^{2} / c^{2}-v^{2} / c^{2}}}\right)_{y}=0 \\
\left(\frac{i u^{2}}{1-u^{2} / c^{2}-v^{2} / c^{2}}+p\right)_{x}+\left(\frac{i u v}{1-u^{2} / c^{2}-v^{2} / c^{2}}\right)_{y}=0 \\
\left(\frac{i u v}{1-u^{2} / c^{2}-v^{2} / c^{2}}\right)_{x}+\left(\frac{i v^{2}}{1-u^{2} / c^{2}-v^{2} / c^{2}}+p\right)_{y}=0
\end{array}\right.
$$

which, taking the Newtonian limit $c \rightarrow+\infty$, turns to the isentropic steady Euler equations (see [1]), where $i=\epsilon+p / c^{2}$ and $\epsilon=m n\left(1+e / c^{2}\right)$ respectively denote the enthalpy per unit volume and the energy per unit volume, $n, m$ and $e$ are the number density of particles per unit volume, the average rest-mass per particle and

* Supported by National Natural Science Foundation of China (11501488), Yunnan Applied Basic Research Projects (2018FD015), the Scientific Research Foundation Project of Yunnan Education Department (2018JS150), the PhD Research Startup Foundation of Yunnan Normal University (2016zb012), Nan Hu Young Scholar Supporting Program of XYNU.

Keywords and phrases: Relativistic Euler equations, steady flow, Riemann problem, delta shock wave, delta contact discontinuity.

1 Department of Mathematics, Yunnan Normal University, Kunming 650500, PR China.

2 College of Mathematics and Statistics, Xinyang Normal University, Xinyang 464000, PR China.

** Corresponding author: yuzhang13120@126.com

(C) The authors. Published by EDP Sciences, 2021 
the internal energy per unit volume, $p$ and $q=(u, v)$ the pressure and velocity in $(x, y)$-plane, $c$ the speed of light.

With the help of Bernoulli's law, the irrotational fluid for (1.1) has been strictly derived and systemically studied by Lai and Shen [8], Luan et al. [11] and Chen et al. [2]. Concretely speaking, the authors in [8] considered the Goursat problems, and mixed initial and boundary value problems. While in [11], the motion of supersonic flow entered into a convex duct was investigated. Besides, the boundary value problems of the 2D steady relativistic Euler equations with general equation of state were solved in [2].

In view of the law of thermodynamics

$$
d\left(\frac{i}{n}\right)=\frac{1}{n} \mathrm{~d} p+T \mathrm{~d} s
$$

where $T$ is the absolute temperature and $s$ the entropy per particle, one can easily deduce that $i=n$, if the fluid is considered as an isentropic and pressureless one, namely $p=0$ and $s$ is constant. In this situation, (1.1) becomes

$$
\left\{\begin{array}{l}
\left(\frac{n u}{\sqrt{1-u^{2} / c^{2}-v^{2} / c^{2}}}\right)_{x}+\left(\frac{n v}{\sqrt{1-u^{2} / c^{2}-v^{2} / c^{2}}}\right)_{y}=0 \\
\left(\frac{n u^{2}}{1-u^{2} / c^{2}-v^{2} / c^{2}}\right)_{x}+\left(\frac{n u v}{1-u^{2} / c^{2}-v^{2} / c^{2}}\right)_{y}=0 \\
\left(\frac{n u v}{1-u^{2} / c^{2}-v^{2} / c^{2}}\right)_{x}+\left(\frac{n v^{2}}{1-u^{2} / c^{2}-v^{2} / c^{2}}\right)_{y}=0
\end{array}\right.
$$

called the two-dimensional steady pressureless isentropic relativistic Euler equations. It is well known that a flow is formed by both the effect of inertia and the effect of pressure difference, so the pressureless equations (1.3) can be regarded as a reduced model of (1.1) by neglecting the effect of pressure difference. Obviously, in the Newtonian limit $c \rightarrow+\infty$, (1.3) is nothing but the classical two-dimensional steady pressureless isentropic flow (see [3]). Thus, (1.3) can be also viewed as the generalization of the classical two-dimensional steady pressureless isentropic flow in the relativistic version.

It is worth mentioning that the Riemann problem and interaction of waves for (1.3) has been recently discussed by Zhang and Zhang [24]. It is shown that Dirac delta function develops in the state variable $n$, the number density of particles. That is to say, the delta shock wave is discovered in the Riemann solutions of (1.3). Generally speaking, the delta shock wave is a kind of nonclassical nonlinear wave, on which at least one of the state variables becomes a singular measure, which is usually used to describe the process of concentration of mass [4] and express the formation of large-scale structures in the universe [13]. Currently, the delta shock wave has been a hot topic of conservation laws and attracted widespread attention of mathematicians and physicists. For example, the Riemann problem for the $3 \times 3$ full relativistic Euler system with generalized Chaplygin proper energy density-pressure relation has been studied by Shao [15], and the delta shock waves on which both two state variables simultaneously contain the Dirac delta function are obtained.

In the study of delta shock wave, an interesting and meaningful problem is the Radon measure initial data problem, which is a special kind of Riemann problem with the initial data containing Dirac measure. In fact, the Radon measure initial data problem has been systemically studied in $[4,7,10,18,21,22,25,26]$ for the zero-pressure type equations of gas dynamics. Readers of interest may also refer to Wang and Zhang [19] for the Chaplygin gas equations, Shen [16] for the pressureless Euler system with friction and Guo et al. [5] for the one-dimensional Chaplygin gas equations with source term, etc. Particularly, the Radon measure initial data problem for the zero-pressure relativistic Euler equations and the isentropic relativistic Chaplygin Euler equations have been studied by Shao and $\mathrm{Li}$ in $[9,14]$. However, to our knowledge, this kind of problem has not 
been discussed in the study of steady relativistic flow fluids, though it has been extensively studied in many non-relativistic fluids and non-steady relativistic equations.

In view of that, we in this paper aim at studying the Radon measure initial data problem for (1.3). For this purpose, we consider the Riemann problem of (1.3) with the initial data containing a Dirac measure, that is

$$
(n, u, v)(0, y)= \begin{cases}\left(n_{-}, u_{-}, v_{-}\right), & y<0 \\ \left(w_{0} \delta, u_{0}, v_{0}\right), & y=0 \\ \left(n_{+}, u_{+}, v_{+}\right), & y>0\end{cases}
$$

in which $n_{i}, u_{i}$ and $v_{i}(i= \pm, 0)$ are arbitrary constants, $\delta$ the standard Dirac delta function, and all the flow states are specially given at the initial data line $x=0$.

In order to establish the global existence of generalized solutions for the Radon measure initial data problem (1.3) and (1.4), an effective way is to consider the perturbation of Riemann initial data

$$
(n, u, v)(0, y)= \begin{cases}\left(n_{-}, u_{-}, v_{-}\right), & -\infty<y<-\epsilon \\ \left(\frac{w_{0}}{2 \epsilon}, u_{0}, v_{0}\right), & -\epsilon<y<\epsilon \\ \left(n_{+}, u_{+}, v_{+}\right), & \epsilon<y<+\infty\end{cases}
$$

for (1.3), where the perturbed parameter $\epsilon>0$ is sufficiently small. Then, by taking $\epsilon \rightarrow 0^{+}$, one obtains the solutions for the system (1.3) with delta initial data (1.4) under the stability theory of weak solutions, which is actually what we need to do. This technique has also been used in $[7,19,26]$, etc.

By analyzing the limit behavior of solutions to the perturbed Riemann problem (1.3) and (1.5) as $\epsilon \rightarrow 0^{+}$, the delta contact discontinuity, on which the state variable $n$ contains the Dirac delta function, is obtained in the solutions of the steady pressureless isentropic relativistic Euler equations (1.3) with delta initial data (1.4). The delta contact discontinuity, generally speaking, is a kind of nonclassical hyperbolic wave, which is a Dirac delta function supported on a contact discontinuity. It has already appeared in the magnetohydrodynamics equations [12] and the nonlinear chromatography equations [6, 17, 23], etc. However, no results are found on this topic to the relativistic steady flow so far.

This paper is organized as follows. In Section 2, we review Riemann solutions for (1.1) with constant initial data of two piecewise. In Section 3, the perturbed Riemann problem (1.1) and (1.5) is solved, and the global structures of solutions to the Radon measure initial data problem (1.1) and (1.4) are established by letting $\epsilon \rightarrow 0^{+}$.

\section{The RiEMAnN PROBLEM With CONSTANT INITIAL DATA}

This section solves the Riemann problem for (1.3) with constant initial data

$$
(n, u, v)(0, y)= \begin{cases}\left(n_{-}, u_{-}, v_{-}\right), & y<0 \\ \left(n_{+}, u_{+}, v_{+}\right), & y>0\end{cases}
$$

Just for convenience, we only consider the case when $x \geq 0$ and $u>0$, since the rest cases, such as $x \geq 0$ and $u<0, x \leq 0$ and $u>0, x \leq 0$ and $u<0$, can be similarly treated. Please see [24] for details.

The system (1.3) has a triple eigenvalue and only two right eigenvectors

$$
\lambda=\frac{v}{u}, \quad \overrightarrow{r_{1}}=(1,0,0)^{T}, \quad \overrightarrow{r_{2}}=(0, u, v)^{T}
$$




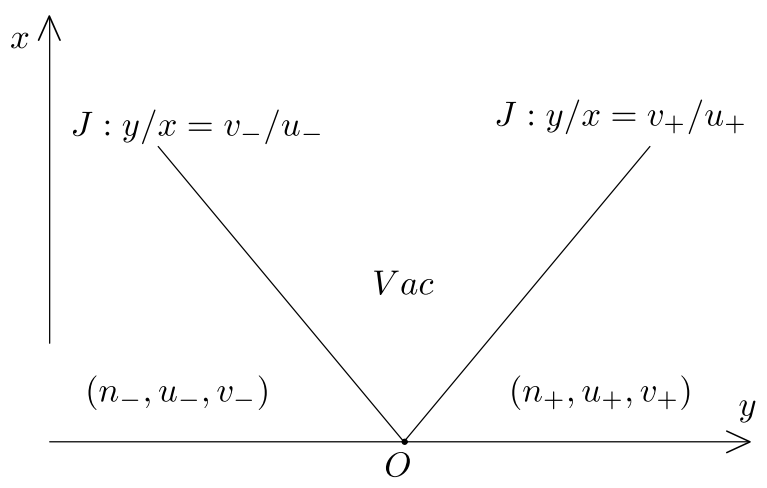

FiguRe 1. Vacuum solution for (1.3) and (2.1).

Since it is easily verified that $\nabla \lambda \cdot \overrightarrow{r_{i}} \equiv 0$ for $i=1,2$, so $\lambda$ is linearly degenerate and the elementary waves involve only contact discontinuities.

As usual, we seek the self-similar solution $(n, u, v)(\xi)(\xi=y / x)$. Then, the Riemann solutions of (1.3) and (2.1) can be constructed by two cases.

When $v_{-} / u_{-} \leq v_{+} / u_{+}$, the vacuum generates. Thus, the solution consists of two contact discontinuities plus a vacuum state besides two constants, which is

$$
(n, u, v)(\xi)= \begin{cases}\left(n_{-}, u_{-}, v_{-}\right), & -\infty<\xi<v_{-} / u_{-}, \\ (0, u(\xi), v(\xi)), & v_{-} / u_{-} \leq \xi \leq v_{+} / u_{+} \\ \left(n_{+}, u_{+}, v_{+}\right), & v_{+} / u_{+}<\xi<+\infty\end{cases}
$$

as shown in Figure 1, where $u(\xi)$ and $v(\xi)$ are two smooth functions satisfying

$$
u\left(\frac{v_{-}}{u_{-}}\right)=u_{-}, \quad u\left(\frac{v_{+}}{u_{+}}\right)=u_{+} \text {and } v\left(\frac{v_{-}}{u_{-}}\right)=v_{-}, \quad v\left(\frac{v_{+}}{u_{+}}\right)=v_{+} .
$$

However, for the case $v_{-} / u_{-}>v_{+} / u_{+}$, the linearly degenerate characteristic lines from $y$-axis must overlap in the domain $\left\{(x, y) \mid v_{+} / u_{+} \leq y / x \leq v_{-} / u_{-}, x \geq 0\right\}$ in $(x, y)$-plane, so the singularity of solutions must develop in this region. As in [3, 20], the delta-shock solution including a weighted Dirac $\delta$-function supported on a curve should be proposed. Thus, we introduce the following definition of a measure solution and recall the definition of two-dimensional weighted delta function.

Definition 2.1. ([24]) A triple distribution $(n, u, v)$ consists of a solution of (1.3) in the sense of measures if it satisfies

(a) $n \in L^{\infty}\left([0, \infty), B M\left(R^{1}\right)\right) \cap C\left([0, \infty), \mathbf{H}^{-s}\left(R^{1}\right)\right)$,

(b) $u \in L^{\infty}\left([0, \infty), L^{\infty}\left(R^{1}\right)\right) \cap C\left([0, \infty), \mathbf{H}^{-s}\left(R^{1}\right)\right)$,

(c) $v \in L^{\infty}\left([0, \infty), L^{\infty}\left(R^{1}\right)\right) \cap C\left([0, \infty), \mathbf{H}^{-s}\left(R^{1}\right)\right)$,

(d) $u$ and $v$ are measurable with respect to $n$ at almost for all $x \geq 0$, 
and

$$
\left\{\begin{array}{l}
\int_{0}^{\infty} \int_{R^{1}}\left(\frac{u}{\sqrt{1-u^{2} / c^{2}-v^{2} / c^{2}}} \phi_{x}+\frac{v}{\sqrt{1-u^{2} / c^{2}-v^{2} / c^{2}}} \phi_{y}\right) \mathrm{d} n \mathrm{~d} x=0 \\
\int_{0}^{\infty} \int_{R^{1}} u\left(\frac{u}{1-u^{2} / c^{2}-v^{2} / c^{2}} \phi_{x}+\frac{v}{1-u^{2} / c^{2}-v^{2} / c^{2}} \phi_{y}\right) \mathrm{d} n \mathrm{~d} x=0 \\
\int_{0}^{\infty} \int_{R^{1}} v\left(\frac{u}{1-u^{2} / c^{2}-v^{2} / c^{2}} \phi_{x}+\frac{v}{1-u^{2} / c^{2}-v^{2} / c^{2}} \phi_{y}\right) \mathrm{d} n \mathrm{~d} x=0
\end{array}\right.
$$

hold in the sense of measures for all test functions $\phi \in C\left([0, \infty) \times R^{1}\right)$.

Definition 2.2. A two-dimensional weighted delta function $w(s) \delta_{S}$ supported on a smooth curve $L$ parameterized as $x=x(s), y=y(s)(a \leq s \leq b)$ is defined by

$$
\left\langle w(s) \delta_{L}, \phi(x, y)\right\rangle=\int_{a}^{b} w(s) \phi(x(s), y(s)) \mathrm{d} s
$$

for all $\phi \in C_{0}^{\infty}\left(R^{2}\right)$.

By these two definitions, one can look for the solution of (1.3) with discontinuity $y=y(x)$ of the form

$$
(n, u, v)(x, y)= \begin{cases}\left(n_{-}, u_{-}, v_{-}\right), & y<y(x), \\ \left(w(x) \delta(y-y(x)), u_{\delta}(x), v_{\delta}(x)\right), & y=y(x), \\ \left(n_{+}, u_{+}, v_{+}\right), & y>y(x)\end{cases}
$$

in which $\left(n_{ \pm}, u_{ \pm}, v_{ \pm}\right)(x, y)$ are piecewise smooth solutions of $(1.3), y(x) \in C^{1}, \delta(\cdot)$ is the standard Dirac measure.

Then, one can prove that (2.7) is a solution of (1.3) in the sense of measures if it satisfies the generalized Rankine-Hugoniot relation

$$
\left\{\begin{aligned}
& \frac{\mathrm{d} y}{\mathrm{~d} x}=\sigma \\
& \frac{\mathrm{d}}{\mathrm{d} x}\left(\frac{w u_{\delta}}{\sqrt{1-u_{\delta}^{2} / c^{2}-v_{\delta}^{2} / c^{2}}}\right)=-\left[\frac{n u}{\sqrt{1-u^{2} / c^{2}-v^{2} / c^{2}}}\right] \sigma+\left[\frac{n v}{\sqrt{1-u^{2} / c^{2}-v^{2} / c^{2}}}\right] \\
& \frac{n u_{\delta}^{2}}{\mathrm{~d} x}\left(\frac{n u v}{1-u_{\delta}^{2} / c^{2}-v_{\delta}^{2} / c^{2}}\right)=-\left[\frac{n u^{2}}{1-u^{2} / c^{2}-v^{2} / c^{2}}\right] \sigma+\left[\frac{n u v}{1-u^{2} / c^{2}-v^{2} / c^{2}}\right] \\
& \frac{\mathrm{d}}{\mathrm{d} x}\left(\frac{w v_{\delta} v_{\delta}}{1-u_{\delta}^{2} / c^{2}-v_{\delta}^{2} / c^{2}}\right)=-\left[\frac{n v^{2}}{1-u^{2} / c^{2}-v^{2} / c^{2}}\right] \sigma+\left[\frac{n v^{2} / c^{2}-v^{2}}{1-u^{2}}\right]
\end{aligned}\right.
$$

with

$$
\sigma=\frac{v_{\delta}}{u_{\delta}}
$$

in which the jump of $G$ across the discontinuity is denoted by $[G]=G_{-}-G_{+}$, and the Lax entropy condition $\lambda\left(n_{+}, u_{+}, v_{+}\right)<\sigma<\lambda\left(n_{+}, u_{-}, v_{-}\right)$, which ensures the uniqueness of solutions and in this situation takes the 


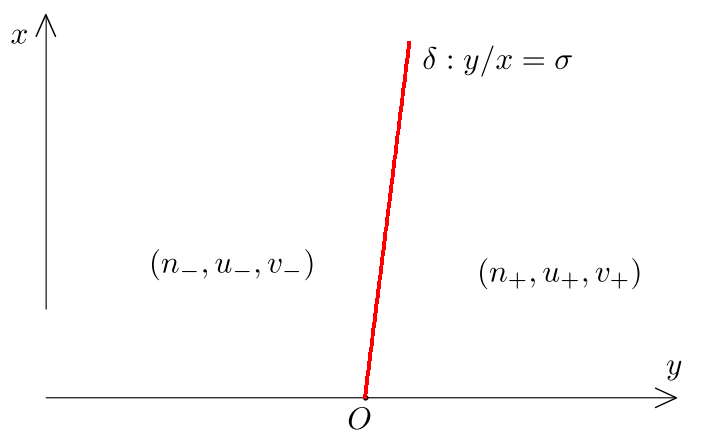

FIGURE 2. Delta-shock solution for (1.3) and (2.1).

form

$$
\frac{v_{+}}{u_{+}}<\frac{v_{\delta}}{u_{\delta}}<\frac{v_{-}}{u_{-}}
$$

A discontinuity satisfying the generalized Rankine-Hugoniot relation (2.8)-(2.9) and the entropy condition (2.10) is called a delta shock wave of (1.3), symbolized by $\delta$.

Under the entropy condition (2.10), by a routine but tedious calculation, we solve the generalized RankineHugoniot relation (2.8) and (2.9) with the condition

$$
x=0: \quad y(0)=0, \quad w(0)=0
$$

to obtain that

$$
\left\{\begin{array}{l}
y(x)=\frac{\sqrt{\frac{n_{-}}{1-u_{-}^{2} / c^{2}-v_{-}^{2} / c^{2}}} v_{-}+\sqrt{\frac{n_{+}}{1-u_{+}^{2} / c^{2}-v_{+}^{2} / c^{2}}} v_{+}}{\sqrt{\frac{n_{-}}{1-u_{-}^{2} / c^{2}-v_{-}^{2} / c^{2}}} u_{-}+\sqrt{\frac{n_{+}}{1-u_{+}^{2} / c^{2}-v_{+}^{2} / c^{2}}} u_{+}} x \\
w(x)=\frac{\sqrt{\frac{n_{-}}{1-u_{-}^{2} / c^{2}-v_{-}^{2} / c^{2}}} \sqrt{\frac{n_{+}}{1-u_{+}^{2} / c^{2}-v_{+}^{2} / c^{2}}}\left(\sqrt{n_{-}}+\sqrt{n_{+}}\right)^{2}}{\left(\sqrt{\frac{n_{-}}{1-u_{-}^{2} / c^{2}-v_{-}^{2} / c^{2}}} u_{-}+\sqrt{\frac{n_{+}}{1-u_{+}^{2} / c^{2}-v_{+}^{2} / c^{2}}} u_{+}\right)^{2}\left(u_{+} v_{-}-u_{-} v_{+}\right) x} \\
u_{\delta}=\frac{A}{\sqrt{1+A^{2} / c^{2}+B^{2} / c^{2}}}, \\
v_{\delta}=\frac{B}{\sqrt{1+A^{2} / c^{2}+B^{2} / c^{2}}},
\end{array}\right.
$$

with $A, B$ are two constants depending on $\left(n_{ \pm}, u_{ \pm}, v_{ \pm}\right)$expressed by

$$
\left\{\begin{array}{c}
A=\frac{\sqrt{\frac{n_{-}}{1-u_{-}^{2} / c^{2}-v_{-}^{2} / c^{2}}} u_{-}+\sqrt{\frac{n_{+}}{1-u_{+}^{2} / c^{2}-v_{+}^{2} / c^{2}}} u_{+}}{\sqrt{n_{-}}+\sqrt{n_{+}}}, \\
B=\frac{\sqrt{\frac{n_{-}}{1-u_{-}^{2} / c^{2}-v_{-}^{2} / c^{2}}} v_{-}+\sqrt{\frac{n_{+}}{1-u_{+}^{2} / c^{2}-v_{+}^{2} / c^{2}}} v_{+}}{\sqrt{n_{-}}+\sqrt{n_{+}}},
\end{array}\right.
$$

which is just the delta-shock solution for (1.3) and (2.1), as shown in Figure 2. 


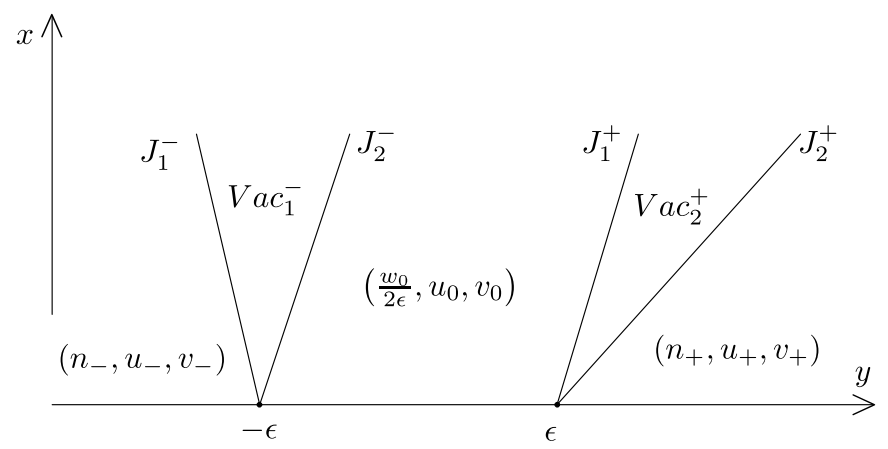

Figure 3. Perturbation analysis for $v_{-} / u_{-}<v_{0} / u_{0}<v_{+} / u_{+}$.

Remark 2.3. So far, we have obtained the Riemann solution of (1.3) and (2.1). It is noticed that, in the non-relativistic limit $(c \rightarrow+\infty)$, the delta-shock solution shown in (2.12) and (2.13) is consistent with that of the steady pressureless isentropic flow (see [3]).

\section{Riemann PROBlem With DELTA Initial DATA}

In this section, we are dedicating to solving the Riemann problem (1.4) for (1.3). To this end, we at first establish the configuration of Riemann solution for (1.3) with (1.5). Base on this, by letting $\epsilon \rightarrow 0^{+}$, we obtain the solution of (1.3) and (1.4) immediately with the help of the stability theory of weak solutions.

For the Riemann problem (1.3) and (1.5), it requires to discuss the interaction of waves. To make sure that all the cases are covered completely, we divide our discussion into three situations according to the different combinations of waves from $(-\epsilon, 0)$ and $(\epsilon, 0)$, which corresponds to the different combinations of $v_{-} / u_{-}, v_{0} / u_{0}$ and $v_{+} / u_{+}$.

Now, let us consider them case by case.

Case 3.1. $v_{-} / u_{-} \leq v_{0} / u_{0} \leq v_{+} / u_{+}$.

This case is divided into three subcases.

Subcase 3.1.1. $v_{-} / u_{-}<v_{0} / u_{0}<v_{+} / u_{+}$.

As shown in Figure 3, when $x$ is small enough, the solution of the initial value problem (1.3) and (1.5) can be expressed briefly as

$$
\left(n_{-}, u_{-}, v_{-}\right)+J_{1}^{-}+V a c_{1}^{-}+J_{2}^{-}+\left(\frac{w_{0}}{2 \epsilon}, u_{0}, v_{0}\right)+J_{1}^{+}+V a c_{2}^{+}+J_{2}^{+}+\left(n_{+}, u_{+}, v_{+}\right),
$$

where "+" means "followed by", or explicitly formulated as

$$
(n, u, v)(x, y)= \begin{cases}\left(n_{-}, u_{-}, v_{-}\right), & y<y_{1}^{-}(x), \\ V a c_{1}^{-}, & y_{1}^{-}(x) \leq y \leq y_{2}^{-}(x), \\ \left(\frac{w_{0}}{2 \epsilon}, u_{0}, v_{0}\right), & y_{2}^{-}(x)<y<y_{1}^{+}(x), \\ V a c_{2}^{+}, & y_{1}^{+}(x) \leq y \leq y_{2}^{+}(x), \\ \left(n_{+}, u_{+}, v_{+}\right), & y>y_{2}^{+}(x),\end{cases}
$$


where $y_{1}^{-}(x)=\frac{v_{-}}{u_{-}} x-\epsilon, y_{2}^{-}(x)=\frac{v_{0}}{u_{0}} x-\epsilon, y_{1}^{+}(x)=\frac{v_{0}}{u_{0}} x+\epsilon$ and $y_{1}^{+}(x)=\frac{v_{+}}{u_{+}} x+\epsilon$ denote the position of the contact discontinuities $J_{1}^{-}, J_{2}^{-}, J_{1}^{+}$and $J_{2}^{+}$, respectively, $V a c_{1}^{-}$and $V a c_{2}^{+}$denote vacuum solution $\left(0, u_{1}(x, y), v_{1}(x, y)\right)$ and $\left(0, u_{2}(x, y), v_{2}(x, y)\right)$ with $u_{i}(x, y)$ and $v_{i}(x, y)(i=1,2)$ are arbitrary smooth functions satisfying

$$
\begin{array}{llll}
u_{1}\left(x, y_{1}^{-}(x)\right)=u_{-}, & v_{1}\left(x, y_{1}^{-}(x)\right)=v_{-}, & u_{1}\left(x, y_{2}^{-}(x)\right)=u_{0}, & v_{1}\left(x, y_{2}^{-}(x)\right)=v_{0}, \\
u_{2}\left(x, y_{1}^{+}(x)\right)=u_{0}, & v_{2}\left(x, y_{1}^{+}(x)\right)=v_{0}, & u_{2}\left(x, y_{2}^{+}(x)\right)=u_{+}, & v_{2}\left(x, y_{2}^{+}(x)\right)=v_{+} .
\end{array}
$$

Noting that both the propagation speeds of the contact discontinuities $J_{1}^{+}$and $J_{2}^{-}$are $v_{0} / u_{0}$, so $J_{2}^{-}$will never overtake $J_{1}^{+}$and there is no collision of waves.

For any given $x \geq 0$, one can deduce that

$$
\lim _{\epsilon \rightarrow 0^{+}} y_{1}^{-}(x)=\frac{v_{-}}{u_{-}} x:=\widetilde{y_{1}^{-}}(x), \quad \lim _{\epsilon \rightarrow 0^{+}} y_{2}^{+}(x)=\frac{v_{+}}{u_{+}} x:=\widetilde{y_{2}^{+}}(x)
$$

and

$$
\lim _{\epsilon \rightarrow 0^{+}} y_{1}^{+}(x)=\lim _{\epsilon \rightarrow 0^{+}} y_{2}^{-}(x)=\frac{v_{0}}{u_{0}} x:=\widetilde{y}(x),
$$

as well as

$$
\lim _{\epsilon \rightarrow 0^{+}} n=\lim _{\epsilon \rightarrow 0^{+}} \frac{w_{0}}{2 \epsilon}=+\infty, \quad \lim _{\epsilon \rightarrow 0^{+}} \int_{y_{2}^{-}(x)}^{y_{1}^{+}(x)} n \mathrm{~d} y=\lim _{\epsilon \rightarrow 0^{+}} \int_{\frac{u_{0}}{v_{0}} x-\epsilon}^{\frac{u_{0}}{v_{0}} x+\epsilon} \frac{w_{0}}{2 \epsilon} \mathrm{d} y=w_{0} .
$$

From (3.3), we see that the two contact discontinuities $J_{2}^{-}$and $J_{1}^{+}$coincide to be a new contact discontinuity as $\epsilon \rightarrow 0^{+}$. Furthermore, it is observed from (3.4) that the coincidence of $J_{2}^{-}$and $J_{1}^{+}$in fact leads to a new type of nonlinear hyperbolic wave, namely, a delta contact discontinuity, denoted by $\delta J: y=\widetilde{y}(x)$, which is a Dirac delta function supported on a contact discontinuity. Here, this weighed Dirac delta function comes from the singularity of the number density $n$ when $\epsilon \rightarrow 0^{+}$. For more demonstrations about delta contact discontinuity, we refer to $[6,12,17,23]$, etc.

The speed, location and weight of the delta contact discontinuity $\delta J$ are given by

$$
\frac{\mathrm{d} y}{\mathrm{~d} x}=\frac{v_{0}}{u_{0}}, \quad y(x)=\frac{v_{0}}{u_{0}} x, \quad w(x)=w_{0} \quad \text { for } \quad x \geq 0 .
$$

Moreover, we verify that $\delta J$ satisfies generalized Rankine-Hugoniot condition (2.8).

Thereby, as shown in Figure 4, the solution for (1.3) and (1.4) can be formulated by

$$
\left(n_{-}, u_{-}, v_{-}\right)+J_{1}+V a c_{1}+\delta J+V a c_{2}+J_{2}+\left(n_{+}, u_{+}, v_{+}\right),
$$




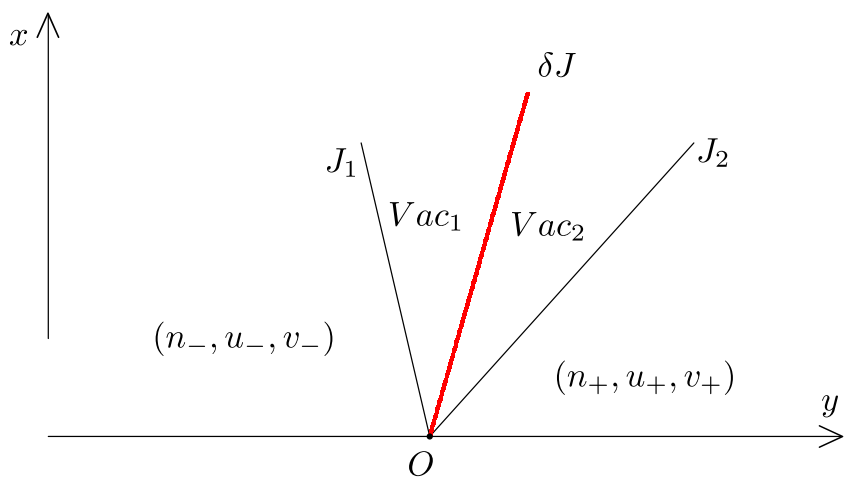

FiguRE 4. Solution for $v_{-} / u_{-}<v_{0} / u_{0}<v_{+} / u_{+}$.

or explicitly expressed as

$$
(n, u, v)(x, y)= \begin{cases}\left(n_{-}, u_{-}, v_{-}\right), & y<\widetilde{y_{1}^{-}}(x) \\ V a c_{1}, & \widetilde{y_{1}^{-}}(x) \leq y \leq \widetilde{y}(x), \\ \left(w_{0} \delta(y-\tilde{y}(x)), u_{0}, v_{0}\right), & y=\tilde{y}(x), \\ V a c_{2}, & \tilde{y}(x) \leq y \leq \widetilde{y_{2}^{+}}(x), \\ \left(n_{+}, u_{+}, v_{+}\right), & y>\widetilde{y_{2}^{+}}(x),\end{cases}
$$

where $\tilde{y}(x)$ and $w_{0}$ are position and weight of the delta contact discontinuity $\delta J, u_{0}$ and $v_{0}$ the values of $u$ and $v$ on this discontinuity, $\widetilde{y_{1}^{-}}(x)$ and $\widetilde{y_{2}^{+}}(x)$ the position of $J_{1}$ and $J_{2}$, respectively, $V a c_{1}$ and $V a c_{2}$ are vacuum solutions $\left(0, u_{1}(x, y), v_{1}(x, y)\right)$ and $\left(0, u_{2}(x, y), v_{2}(x, y)\right)$ with $u_{i}(x, y)$ and $v_{i}(x, y)(i=1,2)$ are arbitrary smooth functions satisfying

$$
u_{1}\left(x, \widetilde{y_{1}^{-}}(x)\right)=u_{-}, \quad v_{1}\left(x, \widetilde{y_{1}^{-}}(x)\right)=v_{-}, \quad u_{1}\left(x, \widetilde{y_{2}^{+}}(x)\right)=u_{+}, \quad v_{1}\left(x, \widetilde{y_{2}^{+}}(x)\right)=v_{+} .
$$

Subcase 3.1.2. $v_{-} / u_{-}=v_{0} / u_{0}<v_{+} / u_{+}$(If $v_{-} / u_{-}<v_{0} / u_{0}=v_{+} / u_{+}$, then the structure of the solution is similar.)

By a similar discussion as in Subcase 3.1.1, the solution for (1.3) and (1.4) is expressed as

$$
\left(n_{-}, u_{-}, v_{-}\right)+\delta J+V a c_{2}+J_{2}+\left(n_{+}, u_{+}, v_{+}\right),
$$

namely,

$$
(n, u, v)(x, y)= \begin{cases}\left(n_{-}, u_{-}, v_{-}\right), & y<\tilde{y}(x), \\ \left(w_{0} \delta(y-\tilde{y}(x)), u_{0}, v_{0}\right), & y=\tilde{y}(x), \\ V a c_{2}, & \tilde{y}(x) \leq y \leq \widetilde{y_{2}^{+}}(x), \\ \left(n_{+}, u_{+}, v_{+}\right), & y>\widetilde{y_{2}^{+}}(x),\end{cases}
$$

as shown in Figure 5. The detailed process of constructing solution is omitted here. 


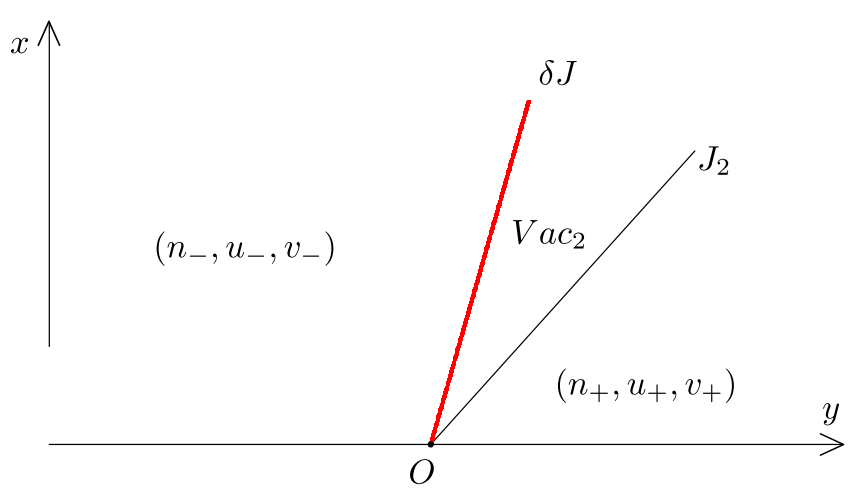

FiguRE 5. Solution for $v_{-} / u_{-}=v_{0} / u_{0}<v_{+} / u_{+}$.

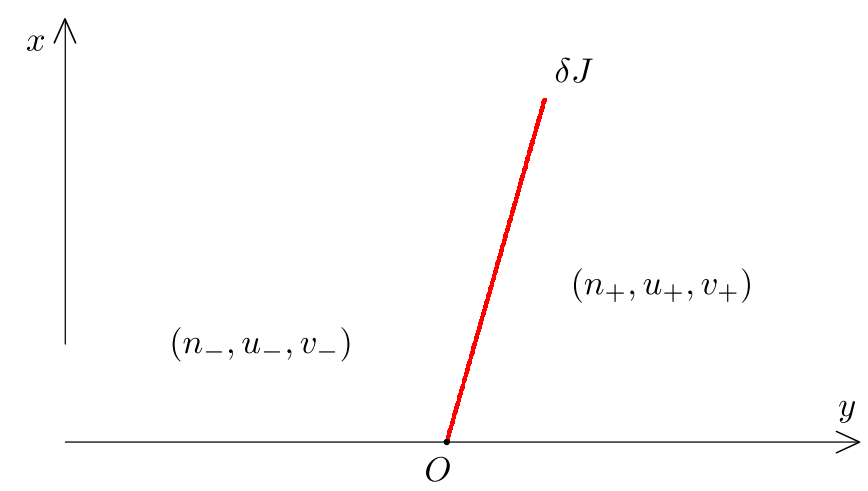

FigURE 6. Solution for $v_{-} / u_{-}=v_{0} / u_{0}=v_{+} / u_{+}$.

Subcase 3.1.3. $v_{-} / u_{-}=v_{0} / u_{0}=v_{+} / u_{+}$.

As presented in Figure 6, in this situation, the solution for (1.3) and (1.4) involves only a delta contact discontinuity and can be symbolized as

$$
\left(n_{-}, u_{-}, v_{-}\right)+\delta J+\left(n_{+}, u_{+}, v_{+}\right)
$$

which is,

$$
(n, u, v)(x, y)= \begin{cases}\left(n_{-}, u_{-}, v_{-}\right), & y<\tilde{y}(x), \\ \left(w_{0} \delta(y-\tilde{y}(x)), u_{0}, v_{0}\right), & y=\tilde{y}(x), \\ \left(n_{+}, u_{+}, v_{+}\right), & y>\tilde{y}(x)\end{cases}
$$

The detailed analysis is omitted.

Case 3.2. $v_{0} / u_{0}<v_{ \pm} / u_{ \pm}$(If $v_{ \pm} / u_{ \pm}<v_{0} / u_{0}$, then the structure of the solution is similar.) 


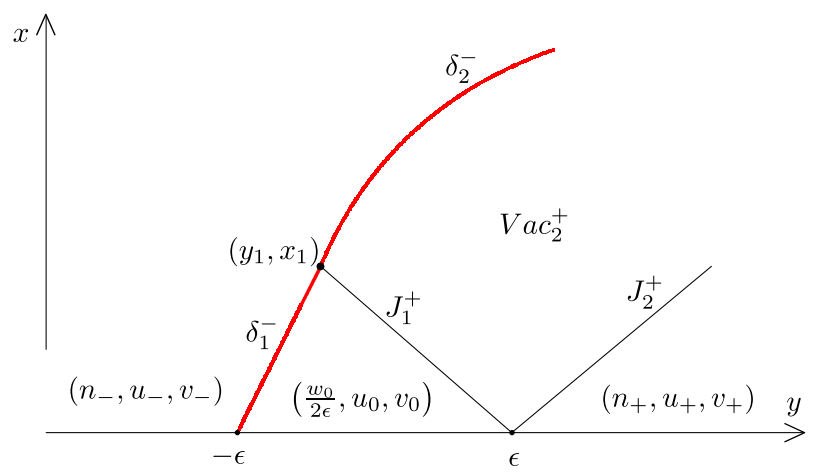

FiguRE 7. Perturbation analysis for $v_{0} / u_{0}<v_{-} / u_{-}<v_{+} / u_{+}$.

Since $v_{0} / u_{0}<v_{ \pm} / u_{ \pm}$, so the solution of Riemann problem (1.3) and (1.5) is denoted by

$$
\left(n_{-}, u_{-}, v_{-}\right)+\delta_{1}^{-}+\left(\frac{w_{0}}{2 \epsilon}, u_{0}, v_{0}\right)+J_{1}^{+}+\operatorname{Vac} c_{2}^{+}+J_{2}^{+}+\left(n_{+}, u_{+}, v_{+}\right)
$$

when $x$ is small enough, which can be written as

$$
(n, u, v)(x, y)= \begin{cases}\left(n_{-}, u_{-}, v_{-}\right), & y<y_{1}(x), \\ \left(w_{1}(x) \delta\left(y-y_{1}(x)\right), u_{\delta_{1}^{-}}, v_{\delta_{1}^{-}}\right), & y=y_{1}(x), \\ \left(\frac{w_{0}}{2 \epsilon}, u_{0}, v_{0}\right), & y_{1}(x)<y<y_{1}^{+}(x), \\ \operatorname{Vac}_{2}^{+}, & y_{1}^{+}(x) \leq y \leq y_{2}^{+}(x), \\ \left(n_{+}, u_{+}, v_{+}\right), & y>y_{2}^{+}(x),\end{cases}
$$

as shown in Figure 7, where the position, weight, and the assignments of $u$ and $v$ for the delta shock wave $\delta_{1}^{-}$ are given as

$$
\left\{\begin{array}{l}
y_{1}(x)=\frac{\sqrt{\frac{n_{-}}{1-u_{-}^{2} / c^{2}-v_{-}^{2} / c^{2}}} v_{-}+\sqrt{\frac{w_{0} / 2 \epsilon}{1-u_{0}^{2} / c^{2}-v_{0}^{2} / c^{2}}} v_{0}}{\sqrt{\frac{n_{-}}{1-u_{-}^{2} / c^{2}-v_{-}^{2} / c^{2}}} u_{-}+\sqrt{\frac{w_{0} / 2 \epsilon}{1-u_{0}^{2} / c^{2}-v_{0}^{2} / c^{2}}} u_{0}} x-\epsilon \\
w_{1}(x)=\frac{\sqrt{\frac{n-}{1-u_{-}^{2} / c^{2}-v_{-}^{2} / c^{2}}} \sqrt{\frac{w_{0} / 2 \epsilon}{1-u_{0}^{2} / c^{2}-v_{0}^{2} / c^{2}}}\left(\sqrt{n_{-}}+\sqrt{w_{0} / 2 \epsilon}\right)^{2}}{\left(\sqrt{\frac{n_{-}}{1-u_{-}^{2} / c^{2}-v_{-}^{2} / c^{2}}} u_{-}+\sqrt{\frac{w_{0} / 2 \epsilon}{1-u_{0}^{2} / c^{2}-v_{0}^{2} / c^{2}}} u_{0}\right)^{2}}\left(u_{0} v_{-}-u_{-} v_{0}\right) x \\
u_{\delta_{1}^{-}}=\frac{A_{\delta_{1}^{-}}}{\sqrt{1+A_{\delta_{1}^{-}}^{2} / c^{2}+B_{\delta_{1}^{-}}^{2} / c^{2}}} \\
v_{\delta_{1}^{-}}=\frac{B_{\delta_{1}^{-}}}{\sqrt{1+A_{\delta_{1}^{-}}^{2} / c^{2}+B_{\delta_{1}^{-}}^{2} / c^{2}}}
\end{array}\right.
$$


with

$$
\left\{\begin{aligned}
& A_{\delta_{1}^{-}}= \frac{\sqrt{\frac{n_{-}}{1-u_{-}^{2} / c^{2}-v_{-}^{2} / c^{2}}} u_{-}+\sqrt{\frac{w_{0} / 2 \epsilon}{1-u_{0}^{2} / c^{2}-v_{0}^{2} / c^{2}}} u_{0}}{\sqrt{n_{-}}+\sqrt{w_{0} / 2 \epsilon}} \\
& B_{\delta_{1}^{-}}=\frac{\sqrt{\frac{n_{-}}{1-u_{-}^{2} / c^{2}-v_{-}^{2} / c^{2}}} v_{-}+\sqrt{\frac{w_{0} / 2 \epsilon}{1-u_{0}^{2} / c^{2}-v_{0}^{2} / c^{2}}} v_{0}}{\sqrt{n_{-}}+\sqrt{w_{0} / 2 \epsilon}}
\end{aligned}\right.
$$

Moreover, $y_{1}^{+}(x)=\frac{v_{0}}{u_{0}} x+\epsilon$ and $y_{2}^{+}(x)=\frac{v_{+}}{u_{+}} x+\epsilon$ are the position of $J_{1}$ and $J_{2}$, respectively, Vac $_{2}^{+}$denotes vacuum solution $\left(0, u_{2}(x, y), v_{2}(x, y)\right)$ with $u_{2}(x, y)$ and $v_{2}(x, y)$ are arbitrary smooth functions satisfying

$$
u_{2}\left(x, y_{1}^{+}(x)\right)=u_{0}, \quad v_{2}\left(x, y_{1}^{+}(x)\right)=v_{0}, \quad u_{2}\left(x, y_{2}^{+}(x)\right)=u_{+}, \quad v_{2}\left(x, y_{2}^{+}(x)\right)=v_{+} .
$$

The propagation speed of $\delta_{1}^{-}$is

$$
\frac{v_{\delta_{1}^{-}}}{u_{\delta_{1}^{-}}}=\frac{\sqrt{\frac{n_{-}}{1-u_{-}^{2} / c^{2}-v_{-}^{2} / c^{2}}} u_{-}+\sqrt{\frac{w_{0} / 2 \epsilon}{1-u_{0}^{2} / c^{2}-v_{0}^{2} / c^{2}}} u_{0}}{\sqrt{\frac{n_{-}}{1-u_{-}^{2} / c^{2}-v_{-}^{2} / c^{2}}} v_{-}+\sqrt{\frac{w_{0} / 2 \epsilon}{1-u_{0}^{2} / c^{2}-v_{0}^{2} / c^{2}}} v_{0}},
$$

which satisfies $v_{0} / u_{0}<v_{\delta_{1}^{-}} / u_{\delta_{1}^{-}}<v_{-} / u_{-}$according to the entropy condition.

Thus, the delta shock wave $\delta_{1}^{-}$must meet the contact discontinuity $J_{1}^{+}$at some point $\left(y_{1}, x_{1}\right)$ determined by

$$
\left\{\begin{aligned}
y_{1}+\epsilon & =\frac{v_{\delta_{1}^{-}}}{u_{\delta_{1}^{-}}} x_{1} \\
y_{1}-\epsilon & =\frac{v_{0}}{u_{0}} x_{1}
\end{aligned}\right.
$$

which solves $y_{1}=y_{1}\left(x_{1}\right)$ with

$$
x_{1}=2 \epsilon \cdot \frac{u_{0}\left(\sqrt{\frac{n_{-}}{1-u_{-}^{2} / c^{2}-v_{-}^{2} / c^{2}}} u_{-}+\sqrt{\frac{w_{0} / 2 \epsilon}{1-u_{0}^{2} / c^{2}-v_{0}^{2} / c^{2}}} u_{0}\right)}{\left(u_{0} v_{-}-u_{-} v_{0}\right) \sqrt{\frac{n_{-}}{1-u_{-}^{2} / c^{2}-v_{-}^{2} / c^{2}}}} .
$$

Now, at $x=x_{1}$, an initial problem for (1.3) with the initial data

$$
(n, u, v)\left(x_{1}, y\right)= \begin{cases}\left(n_{-}, u_{-}, v_{-}\right), & y<y_{1}\left(x_{1}\right), \\ \left(w_{1}\left(x_{1}\right) \delta\left(y-y_{1}\left(x_{1}\right)\right), u_{\delta_{1}^{-}}\left(x_{1}\right), v_{\delta_{1}^{-}}\left(x_{1}\right)\right), & y=y_{1}\left(x_{1}\right), \\ \operatorname{Vac}_{2}^{+}, & y_{1}\left(x_{1}\right) \leq y \leq y_{2}^{+}\left(x_{1}\right) \\ \left(n_{+}, u_{+}, v_{+}\right), & y>y_{2}^{+}\left(x_{1}\right),\end{cases}
$$


is formed. It is solvable by a delta shock wave, denoted by $\delta_{2}^{-}:\left(y_{2}(x), w_{2}(x), u_{\delta_{2}^{-}}(x), v_{\delta_{2}^{-}}(x)\right)$, which obeys the following generalized Rankine-Hugoniot relation

$$
\left\{\begin{array}{l}
\frac{\mathrm{d} y}{\mathrm{~d} x}=\frac{v_{\delta}}{u_{\delta}}, \\
\frac{\mathrm{d}}{\mathrm{d} x}\left(\frac{w u_{\delta}}{\sqrt{1-u_{\delta}^{2} / c^{2}-v_{\delta}^{2} / c^{2}}}\right)=-\frac{n_{-} u_{-}}{\sqrt{1-u_{-}^{2} / c^{2}-v_{-}^{2} / c^{2}}} \cdot \frac{v_{\delta}}{u_{\delta}}+\frac{n_{-} v_{-}}{\sqrt{1-u_{-}^{2} / c^{2}-v_{-}^{2} / c^{2}}} \\
\frac{n_{-} u_{-}^{2}}{\mathrm{~d} x}\left(\frac{w u_{\delta}^{2}}{1-u_{\delta}^{2} / c^{2}-v_{\delta}^{2} / c^{2}}\right)=-\frac{v_{\delta}}{1-u_{-}^{2} / c^{2}-v_{-}^{2} / c^{2}}+\frac{n_{-} u_{-} v_{-}}{1-u_{-}^{2} / c^{2}-v_{-}^{2} / c^{2}} \\
\frac{\mathrm{d}}{\mathrm{d} x}\left(\frac{w u_{\delta} v_{\delta}}{1-u_{\delta}^{2} / c^{2}-v_{\delta}^{2} / c^{2}}\right)=-\frac{n_{-} v_{-}}{1-u_{-}^{2} / c^{2}-v_{-}^{2} / c^{2}} \cdot \frac{v_{\delta}}{u_{\delta}}+\frac{n_{-} v_{-}^{2}}{1-u_{-}^{2} / c^{2}-v_{-}^{2} / c^{2}}
\end{array}\right.
$$

and the initial data

$$
x=x_{1}:\left\{\begin{array}{l}
y\left(x_{1}\right)=y_{1}\left(x_{1}\right):=y_{1} \\
w\left(x_{1}\right)=w_{1}\left(x_{1}\right):=m_{1}, \\
u_{\delta}\left(x_{1}\right)=u_{\delta_{1}^{-}}\left(x_{1}\right):=u_{1} \\
v_{\delta}\left(x_{1}\right)=v_{\delta_{1}^{-}}\left(x_{1}\right):=v_{1}
\end{array}\right.
$$

In what follows, we solve $\left(y_{2}(x), w_{2}(x), u_{\delta_{2}^{-}}(x), v_{\delta_{2}^{-}}(x)\right)$ for $\delta_{2}^{-}$. From (3.15) and (3.16), we have the following

$$
\left\{\begin{array}{l}
\frac{w u_{\delta}}{\sqrt{1-u_{\delta}^{2} / c^{2}-v_{\delta}^{2} / c^{2}}}=-\frac{n_{-} u_{-}\left(y-y_{1}\right)}{\sqrt{1-u_{-}^{2} / c^{2}-v_{-}^{2} / c^{2}}}+\frac{n_{-} v_{-}\left(x-x_{1}\right)}{\sqrt{1-u_{-}^{2} / c^{2}-v_{-}^{2} / c^{2}}}+\frac{m_{1} u_{1}}{\sqrt{1-u_{1}^{2} / c^{2}-v_{1}^{2} / c^{2}}}, \\
\frac{w u_{\delta}^{2}}{1-u_{\delta}^{2} / c^{2}-v_{\delta}^{2} / c^{2}}=-\frac{n_{-} u_{-}^{2}\left(y-y_{1}\right)}{1-u_{-}^{2} / c^{2}-v_{-}^{2} / c^{2}}+\frac{n_{-} u_{-} v_{-}\left(x-x_{1}\right)}{1-u_{-}^{2} / c^{2}-v_{-}^{2} / c^{2}}+\frac{m_{1} u_{1}^{2}}{1-u_{1}^{2} / c^{2}-v_{1}^{2} / c^{2}}, \\
\frac{w u_{\delta} v_{\delta}}{1-u_{\delta}^{2} / c^{2}-v_{\delta}^{2} / c^{2}}=-\frac{n_{-} u_{-} v_{-}\left(y-y_{1}\right)}{1-u_{-}^{2} / c^{2}-v_{-}^{2} / c^{2}}+\frac{n_{-} v_{-}^{2}\left(x-x_{1}\right)}{1-u_{-}^{2} / c^{2}-v_{-}^{2} / c^{2}}+\frac{m_{1} u_{1} v_{1}}{1-u_{1}^{2} / c^{2}-v_{1}^{2} / c^{2}},
\end{array}\right.
$$

where $y=y(x), w=w(x), u_{\delta}=u_{\delta}(x)$ and $v_{\delta}=v_{\delta}(x)$ are the unknowns.

Multiplying the second equation of (3.17) by $v_{\delta} / u_{\delta}$ and subtracting the obtained result into the third one, we have

$$
\begin{gathered}
\frac{n_{-} u_{-}^{2}\left(y-y_{1}\right)}{1-u_{-}^{2} / c^{2}-v_{-}^{2} / c^{2}} \cdot \frac{v_{\delta}}{u_{\delta}}-\frac{n_{-} u_{-} v_{-}\left(y-y_{1}\right)}{1-u_{-}^{2} / c^{2}-v_{-}^{2} / c^{2}}-\frac{n_{-} u_{-} v_{-}\left(x-x_{1}\right)}{1-u_{-}^{2} / c^{2}-v_{-}^{2} / c^{2}} \cdot \frac{v_{\delta}}{u_{\delta}} \\
-\frac{m_{1} u_{1}^{2}}{1-u_{1}^{2} / c^{2}-v_{1}^{2} / c^{2}} \cdot \frac{v_{\delta}}{u_{\delta}}=-\frac{m_{1} u_{1} v_{1}}{1-u_{1}^{2} / c^{2}-v_{1}^{2} / c^{2}}-\frac{n_{-} v_{-}^{2}\left(x-x_{1}\right)}{1-u_{-}^{2} / c^{2}-v_{-}^{2} / c^{2}}
\end{gathered}
$$

or

$$
\begin{aligned}
& \frac{\mathrm{d}}{\mathrm{d} x}\left(\frac{1}{2} \cdot \frac{n_{-} u_{-}^{2}\left(y-y_{1}\right)^{2}}{1-u_{-}^{2} / c^{2}-v_{-}^{2} / c^{2}}-\frac{n_{-} u_{-} v_{-}\left(x-x_{1}\right)\left(y-y_{1}\right)}{1-u_{-}^{2} / c^{2}-v_{-}^{2} / c^{2}}-\frac{m_{1} u_{1}^{2}\left(y-y_{1}\right)}{1-u_{1}^{2} / c^{2}-v_{1}^{2} / c^{2}}\right) \\
= & -\frac{m_{1} u_{1} v_{1}}{1-u_{1}^{2} / c^{2}-v_{1}^{2} / c^{2}}-\frac{n_{-} v_{-}^{2}\left(x-x_{1}\right)}{1-u_{-}^{2} / c^{2}-v_{-}^{2} / c^{2}},
\end{aligned}
$$


which gives that

$$
\begin{aligned}
\frac{1}{2} & \frac{n_{-} u_{-}^{2}}{1-u_{-}^{2} / c^{2}-v_{-}^{2} / c^{2}}\left(y-y_{1}\right)^{2}-\left(\frac{n_{-} u_{-} v_{-}}{1-u_{-}^{2} / c^{2}-v_{-}^{2} / c^{2}}\left(x-x_{1}\right)+\frac{m_{1} u_{1}^{2}}{1-u_{1}^{2} / c^{2}-v_{1}^{2} / c^{2}}\right)\left(y-y_{1}\right) \\
& +\left(\frac{m_{1} u_{1} v_{1}}{1-u_{1}^{2} / c^{2}-v_{1}^{2} / c^{2}}\left(x-x_{1}\right)+\frac{1}{2} \frac{n_{-} v_{-}^{2}}{1-u_{-}^{2} / c^{2}-v_{-}^{2} / c^{2}}\left(x-x_{1}\right)^{2}\right)=0,
\end{aligned}
$$

It is clear that (3.19) can be viewed as a quadratic equation on $\left(y-y_{1}\right)$. Denoted by

$$
\begin{aligned}
\Delta= & \left(\frac{n_{-} u_{-} v_{-}\left(x-x_{1}\right)}{1-u_{-}^{2} / c^{2}-v_{-}^{2} / c^{2}}+\frac{m_{1} u_{1}^{2}}{1-u_{1}^{2} / c^{2}-v_{1}^{2} / c^{2}}\right)^{2} \\
& -4 \times \frac{1}{2} \times \frac{n_{-} u_{-}^{2}}{1-u_{-}^{2} / c^{2}-v_{-}^{2} / c^{2}} \times\left(\frac{m_{1} u_{1} v_{1}\left(x-x_{1}\right)}{1-u_{1}^{2} / c^{2}-v_{1}^{2} / c^{2}}+\frac{1}{2} \frac{n_{-} v_{-}^{2}\left(x-x_{1}\right)^{2}}{1-u_{-}^{2} / c^{2}-v_{-}^{2} / c^{2}}\right) \\
= & \frac{m_{1}^{2} u_{1}^{4}}{\left(1-u_{1}^{2} / c^{2}-v_{1}^{2} / c^{2}\right)^{2}}+\frac{2 n_{-} u_{-} m_{1} u_{1}\left(v_{-} u_{1}-u_{-} v_{1}\right)}{\left(1-u_{1}^{2} / c^{2}-v_{1}^{2} / c^{2}\right)\left(1-u_{-}^{2} / c^{2}-v_{-}^{2} / c^{2}\right)}\left(x-x_{1}\right) .
\end{aligned}
$$

Then, it follows from (3.19) that

$$
y-y_{1}=\frac{\frac{n_{-} u_{-} v_{-}}{1-u_{-}^{2} / c^{2}-v_{-}^{2} / c^{2}}\left(x-x_{1}\right)+\frac{m_{1} u_{1}^{2}}{1-u_{1}^{2} / c^{2}-v_{1}^{2} / c^{2}} \pm \sqrt{\Delta}}{\frac{n_{-} u_{-}^{2}}{1-u_{-}^{2} / c^{2}-v_{-}^{2} / c^{2}}} .
$$

By entropy condition, we chose

$$
y=\frac{\frac{n_{-} u_{-} v_{-}}{1-u_{-}^{2} / c^{2}-v_{-}^{2} / c^{2}}\left(x-x_{1}\right)+\frac{m_{1} u_{1}^{2}}{1-u_{1}^{2} / c^{2}-v_{1}^{2} / c^{2}}-\sqrt{\Delta}}{\frac{n_{-} u_{-}^{2}}{1-u_{-}^{2} / c^{2}-v_{-}^{2} / c^{2}}}+y_{1}
$$

as the admissible solution that suits the physical requirement.

In the following, let us solve $u_{\delta}(x)$ and $v_{\delta}(x)$. It is obtained from the first two equations of (3.17) that

$$
\frac{u_{\delta}}{\sqrt{1-u_{\delta}^{2} / c^{2}-v_{\delta}^{2} / c^{2}}}=\frac{\frac{n_{-} u_{-}^{2}\left(y-y_{1}\right)}{1-u_{-}^{2} / c^{2}-v_{-}^{2} / c^{2}}-\frac{n_{-} u_{-} v_{-}\left(x-x_{1}\right)}{1-u_{-}^{2} / c^{2}-v_{-}^{2} / c^{2}}-\frac{m_{1} u_{1}^{2}}{1-u_{1}^{2} / c^{2}-v_{1}^{2} / c^{2}}}{\frac{n_{-} u_{-}\left(y-y_{1}\right)}{\sqrt{1-u_{-}^{2} / c^{2}-v_{-}^{2} / c^{2}}}-\frac{n_{-} v_{-}\left(x-x_{1}\right)}{\sqrt{1-u_{-}^{2} / c^{2}-v_{-}^{2} / c^{2}}}-\frac{m_{1} u_{1}}{\sqrt{1-u_{1}^{2} / c^{2}-v_{1}^{2} / c^{2}}}} .
$$

Taking (3.21) into (3.22) and by a rather tedious calculation, one has

$$
\frac{u_{\delta}}{\sqrt{1-u_{\delta}^{2} / c^{2}-v_{\delta}^{2} / c^{2}}}=\frac{\frac{u_{-} \sqrt{\Delta}}{\sqrt{1-u_{-}^{2} / c^{2}-v_{-}^{2} / c^{2}}}}{\sqrt{\Delta}-\frac{m_{1} u_{1}}{\sqrt{1-u_{1}^{2} / c^{2}-v_{1}^{2} / c^{2}}}\left(\frac{u_{1}}{\sqrt{1-u_{1}^{2} / c^{2}-v_{1}^{2} / c^{2}}}-\frac{u_{-}}{\sqrt{1-u_{-}^{2} / c^{2}-v_{-}^{2} / c^{2}}}\right)}:=K(x)
$$


Similarly, it follows from the first and third equations of (3.17) that

$$
\frac{v_{\delta}}{\sqrt{1-u_{\delta}^{2} / c^{2}-v_{\delta}^{2} / c^{2}}}=\frac{\frac{n_{-} u_{-} v_{-}\left(y-y_{1}\right)}{1-u_{-}^{2} / c^{2}-v_{-}^{2} / c^{2}}-\frac{n_{-} v_{-}^{2}\left(x-x_{1}\right)}{1-u_{-}^{2} / c^{2}-v_{-}^{2} / c^{2}}-\frac{m_{1} u_{1} v_{1}}{1-u_{1}^{2} / c^{2}-v_{1}^{2} / c^{2}}}{\frac{n_{-} u_{-}\left(y-y_{1}\right)}{\sqrt{1-u_{-}^{2} / c^{2}-v_{-}^{2} / c^{2}}}-\frac{n_{-} v_{-}\left(x-x_{1}\right)}{\sqrt{1-u_{-}^{2} / c^{2}-v_{-}^{2} / c^{2}}}-\frac{m_{1} u_{1}}{\sqrt{1-u_{1}^{2} / c^{2}-v_{1}^{2} / c^{2}}}} .
$$

Inserting (3.21) into (3.24) yields

$$
\frac{v_{\delta}}{\sqrt{1-u_{\delta}^{2} / c^{2}-v_{\delta}^{2} / c^{2}}}=\frac{\frac{m_{1} u_{1}\left(u_{1} v_{-}-v_{1} u_{-}\right)}{\left(1-u_{1}^{2} / c^{2}-v_{1}^{2} / c^{2}\right) \sqrt{1-u_{-}^{2} / c^{2}-v_{-}^{2} / c^{2}}}-\frac{v_{-}}{\sqrt{1-u_{-}^{2} / c^{2}-v_{-}^{2} / c^{2}}} \sqrt{\Delta}}{\frac{m_{1} u_{1}}{\sqrt{1-u_{1}^{2} / c^{2}-v_{1}^{2} / c^{2}}}\left(\frac{u_{1}}{\sqrt{1-u_{1}^{2} / c^{2}-v_{1}^{2} / c^{2}}}-\frac{u_{-}}{\sqrt{1-u_{-}^{2} / c^{2}-v_{-}^{2} / c^{2}}}\right)-\sqrt{\Delta}}:=L(x) .
$$

Then, we get from (3.23) and (3.25) that

$$
u_{\delta}(x)=\frac{K(x)}{\sqrt{1+K^{2}(x) / c^{2}+L^{2}(x) / c^{2}}}, \quad v_{\delta}(x)=\frac{L(x)}{\sqrt{1+K^{2}(x)^{2} / c^{2}+L^{2}(x) / c^{2}}} .
$$

Finally, we solve $w(x)$. From the first equation of (3.17), one has

$$
w(x)=\frac{-\frac{n_{-} u_{-}\left(y-y_{1}\right)}{\sqrt{1-u_{-}^{2} / c^{2}-v_{-}^{2} / c^{2}}}+\frac{n_{-} v_{-}\left(x-x_{1}\right)}{\sqrt{1-u_{-}^{2} / c^{2}-v_{-}^{2} / c^{2}}}+\frac{m_{1} u_{1}}{\sqrt{1-u_{1}^{2} / c^{2}-v_{1}^{2} / c^{2}}}}{u_{\delta}} \cdot \sqrt{1-u_{\delta}^{2} / c^{2}-v_{\delta}^{2} / c^{2}} .
$$

Then, owing to (3.21) and (3.23), by a complex computation, we have

$$
w(x)=\frac{1-u_{\delta}^{2} / c^{2}-v_{\delta}^{2} / c^{2}}{u_{\delta}^{2}} \sqrt{\Delta}=\frac{\sqrt{\Delta}}{K^{2}(x)} .
$$

So far, we have obtained the solution $\left(y(x), w(x), u_{\delta}(x), v_{\delta}(x)\right)$ of the generalized Rankine-Hugoniot relation (3.15) with the initial data (3.16). Thus, we summarize the delta shock wave $\delta_{2}^{-}:\left(y_{2}(x), w_{2}(x), u_{\delta_{2}^{-}}(x), v_{\delta_{2}^{-}}(x)\right)$ as follows

$$
\delta_{2}^{-}:\left\{\begin{array}{l}
y_{2}(x)=\frac{\frac{n_{-} u_{-} v_{-}}{1-u_{-}^{2} / c^{2}-v_{-}^{2} / c^{2}}\left(x-x_{1}\right)+\frac{m_{1} u_{1}^{2}}{1-u_{1}^{2} / c^{2}-v_{1}^{2} / c^{2}}-\sqrt{\Delta}}{\frac{n_{-} u_{-}^{2}}{1-u_{-}^{2} / c^{2}-v_{-}^{2} / c^{2}}}+y_{1}\left(x_{1}\right), \\
w_{2}(x)=\frac{1-u_{\delta}^{2} / c^{2}-v_{\delta}^{2} / c^{2}}{u_{\delta}^{2}} \sqrt{\Delta}=\frac{\sqrt{\Delta}}{K^{2}(x)} \\
u_{\delta_{2}^{-}}(x)=\frac{K(x)}{\sqrt{1+K^{2}(x) / c^{2}+L^{2}(x) / c^{2}}}, \\
v_{\delta_{2}^{-}}(x)=\frac{L(x)}{\sqrt{1+K^{2}(x)^{2} / c^{2}+L^{2}(x) / c^{2}}},
\end{array}\right.
$$

in which $\Delta, K(x)$ and $L(x)$ are given in (3.20), (3.23) and (3.25), respectively.

Therefore, for the small $x$ satisfying $x>x_{1}$, the solution to (1.3) and (1.5) is

$$
\left(n_{-}, u_{-}, v_{-}\right)+\delta_{2}^{-}+\operatorname{Vac}_{2}^{+}+J_{2}^{+}+\left(n_{+}, u_{+}, v_{+}\right) \text {, }
$$


or written as

$$
(n, u, v)(x, y)= \begin{cases}\left(n_{-}, u_{-}, v_{-}\right), & y<y_{2}(x), \\ \left(w_{2}(x) \delta\left(y-y_{2}(x)\right), u_{\delta_{2}^{-}}(x), v_{\delta_{2}^{-}}(x)\right), & y=y_{2}(x), \\ V a c_{2}^{+}, & y_{2}(x) \leq y \leq y_{2}^{+}(x), \\ \left(n_{+}, u_{+}, v_{+}\right), & y>y_{2}^{+}(x),\end{cases}
$$

where $y_{2}(x), w_{2}(x), u_{\delta_{2}^{-}}(x)$ and $v_{\delta_{2}^{-}}(x)$ are shown in (3.28).

In what follows, we need to construct the solution of (1.3) and (1.5) for all $x>x_{1}$. To this end, two subcases should been discussed.

Subcase 3.2.1. $v_{0} / u_{0}<v_{-} / u_{-}<v_{+} / u_{+}$.

By a routine calculation, it can be verified from (3.28) that

$$
\lim _{x \rightarrow+\infty} \frac{v_{\delta_{2}^{-}}(x)}{u_{\delta_{2}^{-}}(x)}=\frac{v_{-}}{u_{-}}<\frac{v_{+}}{u_{+}}
$$

which implies that the delta shock wave $\delta_{2}^{-}$will never penetrate the whole vacuum region, as shown in Figure 7 .

Let us analyze the limit behavior of solutions. As $\epsilon \rightarrow 0^{+}$, from (3.11)-(3.13), one can easily check that

$$
x_{1} \rightarrow 0, \quad y_{1}\left(x_{1}\right) \rightarrow 0, \quad A_{\delta_{1}^{-}} \rightarrow \frac{u_{0}}{\sqrt{1-u_{0}^{2} / c^{2}-v_{0}^{2} / c^{2}}}, \quad B_{\delta_{1}^{-}} \rightarrow \frac{v_{0}}{\sqrt{1-u_{0}^{2} / c^{2}-v_{0}^{2} / c^{2}}}
$$

Then, from (3.10) we immediately obtain

$$
\lim _{\epsilon \rightarrow 0^{+}} u_{\delta_{1}^{-}}=\lim _{\epsilon \rightarrow 0^{+}} u_{1}=\frac{\frac{u_{0}}{\sqrt{1-u_{0}^{2} / c^{2}-v_{0}^{2} / c^{2}}}}{\sqrt{1+\frac{u_{0}^{2} / c^{2}}{1-u_{0}^{2} / c^{2}-v_{0}^{2} / c^{2}}+\frac{v_{0}^{2} / c^{2}}{1-u_{0}^{2} / c^{2}-v_{0}^{2} / c^{2}}}}=u_{0}
$$

and similarly $\lim _{\epsilon \rightarrow 0^{+}} v_{\delta_{1}^{-}}=\lim _{\epsilon \rightarrow 0^{+}} v_{1}=v_{0}$.

Moreover, putting (3.13) into the second one of (3.10) yields that

$$
w_{1}\left(x_{1}\right)=m_{1}=\frac{2 \epsilon \sqrt{\frac{w_{0} /(2 \epsilon)}{1-u_{0}^{2} / c^{2}-v_{0}^{2} / c^{2}}}\left(\sqrt{n_{-}}+\sqrt{w_{0} /(2 \epsilon)}\right)^{2} u_{0}}{\sqrt{\frac{n_{-}}{1-u_{-}^{2} / c^{2}-v_{-}^{2} / c^{2}}} u_{-}+\sqrt{\frac{w_{0} /(2 \epsilon)}{1-u_{0}^{2} / c^{2}-v_{0}^{2} / c^{2}}} u_{0}}
$$

then one can prove that

$$
\lim _{\epsilon \rightarrow 0^{+}} w_{1}\left(x_{1}\right)=\lim _{\epsilon \rightarrow 0^{+}} m_{1}=\lim _{\epsilon \rightarrow 0^{+}} \frac{u_{0} \sqrt{\frac{w_{0} /(2 \epsilon)}{1-u_{0}^{2} / c^{2}-v_{0}^{2} / c^{2}}}\left(2 \epsilon n_{-}+w_{0}+4 \epsilon \sqrt{n_{-}} \sqrt{w_{0} /(2 \epsilon)}\right)}{\sqrt{\frac{n_{-}}{1-u_{-}^{2} / c^{2}-v_{-}^{2} / c^{2}}} u_{-}+\sqrt{\frac{w_{0} /(2 \epsilon)}{1-u_{0}^{2} / c^{2}-v_{0}^{2} / c^{2}}} u_{0}}=w_{0} .
$$


As a result, using (3.30)-(3.32), we check from (3.28) that

$$
\left\{\begin{array}{l}
\lim _{\epsilon \rightarrow 0^{+}} y_{2}(x)=\frac{\frac{n_{-} u_{-} v_{-}}{1-u_{-}^{-} / c^{2}-v_{-}^{2} / c^{2}} x+\frac{w_{0} u_{0}^{2}}{1-u_{0}^{2} / c^{2}-v_{0}^{2} / c^{2}}-\sqrt{\bar{\Delta}}}{\frac{n-u_{-}^{2}}{1-u_{-}^{2} / c^{2}-v_{-}^{2} / c^{2}}}:=\overline{y_{1}}(x), \\
\lim _{\epsilon \rightarrow 0^{+}} w_{2}(x)=\frac{\sqrt{\bar{\Delta}}}{\bar{K}^{2}(x)}:=\overline{w_{1}}(x), \\
\lim _{\epsilon \rightarrow 0^{+}} u_{\delta_{2}^{-}}(x)=\frac{\bar{K}(x)}{\sqrt{1+\bar{K}^{2}(x) / c^{2}+\bar{L}^{2}(x) / c^{2}}}:=\bar{u}_{\delta_{1}}(x), \\
\lim _{\epsilon \rightarrow 0^{+}} v_{\delta_{2}^{-}}(x)=\frac{\bar{L}(x)}{\sqrt{1+\bar{K}^{2}(x)^{2} / c^{2}+\bar{L}^{2}(x) / c^{2}}}:=\bar{v}_{\delta_{1}}(x),
\end{array}\right.
$$

in which

$$
\begin{aligned}
& \bar{\Delta}=\lim _{\epsilon \rightarrow 0^{+}} \Delta=\frac{w_{0}^{2} u_{0}^{4}}{\left(1-u_{0}^{2} / c^{2}-v_{0}^{2} / c^{2}\right)^{2}}+\frac{2 n_{-} u_{-} w_{0} u_{0}\left(v_{-} u_{0}-u_{-} v_{0}\right)}{\left(1-u_{0}^{2} / c^{2}-v_{0}^{2} / c^{2}\right)\left(1-u_{-}^{2} / c^{2}-v_{-}^{2} / c^{2}\right)} x, \\
& \bar{K}(x)=\lim _{\epsilon \rightarrow 0^{+}} K(x)=\frac{\frac{u_{-} \sqrt{\bar{\Delta}}}{\sqrt{1-u_{-}^{2} / c^{2}-v_{-}^{2} / c^{2}}}}{\sqrt{\bar{\Delta}}-\frac{m_{0} u_{0}}{\sqrt{1-u_{0}^{2} / c^{2}-v_{0}^{2} / c^{2}}}\left(\frac{u_{0}}{\sqrt{1-u_{0}^{2} / c^{2}-v_{0}^{2} / c^{2}}}-\frac{u_{-}}{\sqrt{1-u_{-}^{2} / c^{2}-v_{-}^{2} / c^{2}}}\right)}, \\
& \bar{K}(x)=\lim _{\epsilon \rightarrow 0^{+}} L(x)=\frac{\frac{m_{0} u_{0}\left(u_{0} v_{-}-v_{0} u_{-}\right)}{\left(1-u_{0}^{2} / c^{2}-v_{0}^{2} / c^{2}\right) \sqrt{1-u_{-}^{2} / c^{2}-v_{-}^{2} / c^{2}}}-\frac{v_{-}}{\sqrt{1-u_{-}^{2} / c^{2}-v_{-}^{2} / c^{2}}} \sqrt{\bar{\Delta}}}{\frac{m_{0} u_{0}}{\sqrt{1-u_{0}^{2} / c^{2}-v_{0}^{2} / c^{2}}}\left(\frac{u_{0}}{\sqrt{1-u_{0}^{2} / c^{2}-v_{0}^{2} / c^{2}}}-\frac{u_{-}}{\sqrt{1-u_{-}^{2} / c^{2}-v_{-}^{2} / c^{2}}}\right)-\sqrt{\bar{\Delta}}} .
\end{aligned}
$$

Moreover,

$$
\lim _{\epsilon \rightarrow 0^{+}} y_{2}^{+}(x)=\lim _{\epsilon \rightarrow 0^{+}}\left(\frac{v_{+}}{u_{+}} x+\epsilon\right)=\frac{v_{+}}{u_{+}} x:=\widetilde{y^{+}}(x)
$$

Thus, the solution to the steady pressureless isentropic relativistic Euler equations (1.3) with delta initial data (1.4) when $v_{0} / u_{0}<v_{-} / u_{-}<v_{+} / u_{+}$can be constructed as

$$
\left(n_{-}, u_{-}, v_{-}\right)+\delta_{1}+V a c+J+\left(n_{+}, u_{+}, v_{+}\right),
$$

that is,

$$
(n, u, v)(0, y)= \begin{cases}\left(n_{-}, u_{-}, v_{-}\right), & y<\overline{y_{1}}(x), \\ \left(\overline{w_{1}}(x) \delta\left(y-\overline{y_{1}}(x)\right), \bar{u}_{\delta_{1}}(x), \bar{v}_{\delta_{1}}(x)\right), & y=\overline{y_{1}}(x), \\ V a c, & \overline{y_{1}}(x) \leq y \leq \widetilde{y^{+}}(x), \\ \left(n_{+}, u_{+}, v_{+}\right), & y>\widetilde{y^{+}}(x),\end{cases}
$$




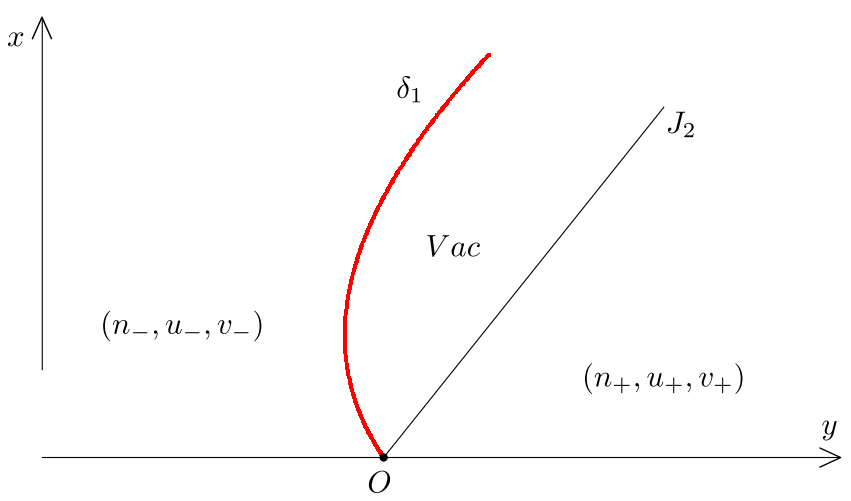

FIGURE 8. Solution for $v_{0} / u_{0}<v_{-} / u_{-}<v_{+} / u_{+}$.

where $\overline{y_{1}}(x), \overline{w_{1}}(x), \bar{u}_{\delta_{1}}(x)$ and $\bar{v}_{\delta_{1}}(x)$, which are given in (3.33), denote the position and weight of the delta shock wave $\delta_{1}$ and the values of $u, v$ on this discontinuity, respectively. Besides, $V a c$ is the vacuum solution $(0, u(x, y), v(x, y))$ with $u(x, y)$ and $v(x, y)$ are arbitrary smooth functions satisfying $u\left(x, \widetilde{y^{+}}(x)\right)=u_{+}$and $v\left(x, \widetilde{y^{+}}(x)\right)=v_{+}$, where $\widetilde{y^{+}}(x)=v_{+} / u_{+}$is the position of the contact discontinuity $J_{2}$, as shown in Figure 8.

Subcase 3.2.2. $v_{0} / u_{0}<v_{+} / u_{+}<v_{-} / u_{-}$.

It is clear that, in this situation, the solution for the steady pressureless isentropic relativistic Euler equations (1.3) with delta initial data (1.4) is the same as that of Subcase 3.2.1 when $x$ is small enough. However, since one can check that

$$
\lim _{x \rightarrow+\infty} \frac{\bar{v}_{\delta_{1}}(x)}{\bar{u}_{\delta_{1}}(x)}=\frac{v_{-}}{u_{-}}>\frac{v_{+}}{u_{+}}
$$

which means that the delta shock wave $\delta_{1}$ must penetrate the whole vacuum region completely and then meet the contact discontinuity $J_{2}$ at some point $\left(y_{2}, x_{2}\right)$, in which $y_{2}=\overline{y_{1}}\left(x_{2}\right)$. Here, the interaction point $\left(y_{2}, x_{2}\right)$ is determined by

$$
\left\{\begin{array}{l}
y=\frac{\frac{n_{-} u_{-} v_{-}}{1-u_{-}^{2} / c^{2}-v_{-}^{2} / c^{2}} x+\frac{w_{0} u_{0}^{2}}{1-u_{0}^{2} / c^{2}-v_{0}^{2} / c^{2}}-\sqrt{\bar{\Delta}}}{\frac{n_{-} u_{-}^{2}}{1-u_{-}^{2} / c^{2}-v_{-}^{2} / c^{2}}}, \\
y=\frac{v_{+}}{u_{+}} x .
\end{array}\right.
$$

Thus, when $x<x_{2}$, the solution of (1.3) and (1.4) for $v_{0} / u_{0}<v_{+} / u_{+}<v_{-} / u_{-}$is the same as that of Subcase 3.2.1, which is expressed in (3.34).

Now, at $x=x_{2}$, a new initial problem for (1.3) with the initial data

$$
(n, u, v)\left(x_{2}, y\right)= \begin{cases}\left(n_{-}, u_{-}, v_{-}\right), & y<\overline{y_{1}}\left(x_{2}\right) \\ \left(\overline{w_{1}}\left(x_{2}\right) \delta\left(y-\overline{y_{1}}\left(x_{2}\right)\right), \bar{u}_{\delta_{1}}\left(x_{2}\right), \bar{v}_{\delta_{1}}\left(x_{2}\right)\right), & y=\overline{y_{1}}\left(x_{2}\right) \\ \left(n_{+}, u_{+}, v_{+}\right), & y>\overline{y_{1}}\left(x_{2}\right)\end{cases}
$$




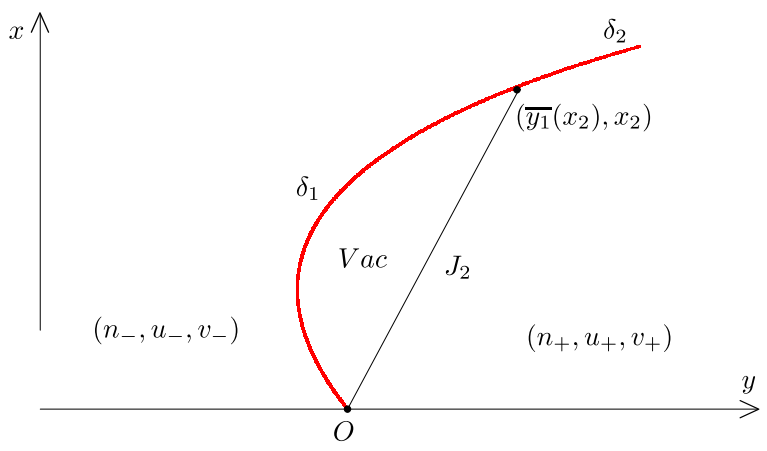

FigURE 9. Solution for $v_{0} / u_{0}<v_{+} / u_{+}<v_{-} / u_{-}$.

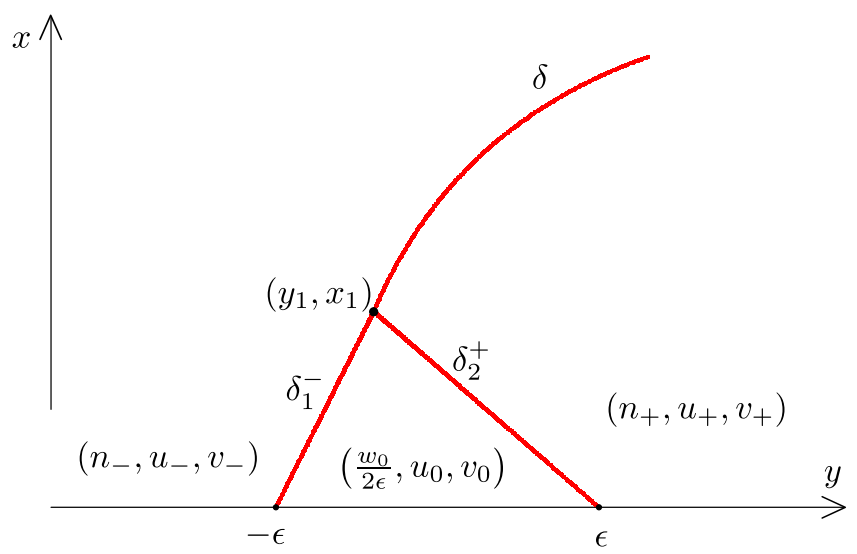

Figure 10. Perturbation analysis for $v_{+} / u_{+}<v_{0} / u_{0}<v_{-} / u_{-}$.

is formed. Obviously, this initial problem is solvable by a new delta shock wave, denoted by $\delta_{2}$, whose position, weight and velocity can be obtained as before, so we omit it here just for brevity. Therefore, in this situation, the solution of (1.3) and (1.4) for $x>x_{2}$ is a delta shock wave, as shown in Figure 9.

Case 3.3. $v_{+} / u_{+}<v_{0} / u_{0}<v_{-} / u_{-}$.

In this situation, the solution of the perturbed Riemann problem (1.3) and (1.5) is

$$
\left(n_{-}, u_{-}, v_{-}\right)+\delta_{1}^{-}+\left(\frac{w_{0}}{2 \epsilon}, u_{0}, v_{0}\right)+\delta_{2}^{+}+\left(n_{+}, u_{+}, v_{+}\right)
$$

when $x$ is small enough, as shown in Figure 10, in which the position and weight of the delta shock wave $\delta_{1}^{-}$ and the assignments of $u$ and $v$ are given in (3.10), and $\delta_{2}^{+}$owns a similar expression.

By entropy condition, we know that the propagation speed of $\delta_{1}^{-}$is $v_{\delta_{1}^{-}} / u_{\delta_{1}^{-}}$, satisfying $v_{0} / u_{0}<v_{\delta_{1}^{-}} / u_{\delta_{1}^{-}}<$ $v_{-} / u_{-}$. While the propagation speed of $\delta_{2}^{+}$is $v_{\delta_{2}^{+}} / u_{\delta_{2}^{+}}$, satisfying $v_{+} / u_{+}<v_{\delta_{2}^{+}} / u_{\delta_{2}^{+}}<v_{0} / u_{0}$. Thus, $\delta_{1}^{-}$must overtake $\delta_{2}^{+}$at the intersection point $\left(y_{1}, x_{1}\right)$, which is

$$
\left(y_{1}, x_{1}\right)=\left(\frac{u_{\delta_{2}} v_{\delta_{1}}+u_{\delta_{1}} v_{\delta_{2}}}{u_{\delta_{2}} v_{\delta_{1}}-u_{\delta_{1}} v_{\delta_{2}}} \epsilon, \frac{2 \epsilon u_{\delta_{1}} u_{\delta_{2}}}{u_{\delta_{2}} v_{\delta_{1}}-u_{\delta_{1}} v_{\delta_{2}}}\right)
$$




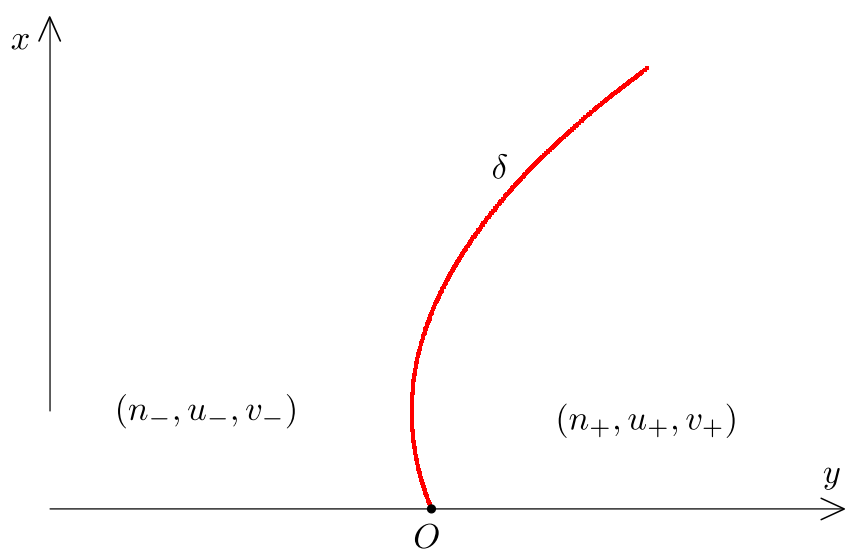

Figure 11. Solution for $v_{+} / u_{+}<v_{0} / u_{0}<v_{-} / u_{-}$.

At $\left(y_{1}, x_{1}\right)$, since $v_{-} / u_{-}>v_{+} / u_{+}$, so a new Riemann problem generates, which is obviously solvable with a new delta shock wave $\delta$. Clearly, the position, weight, and the values of $u, v$ on this delta shock wave can be obtained by solving the generalized Rankine-Hugoniot relation (2.8) with some suitable initial data, as we have done in Case 3.2. The detail is omitted.

Like before, by letting $\epsilon \rightarrow 0^{+}$, one can obtain the solution of (1.3) and (1.4). However, inspired from the discussions for the above two cases, we conclude that the solution of (1.3) and (1.4) consists of a single delta shock wave omitting from the original position, which connects the two constant states $\left(n_{ \pm}, u_{ \pm}, v_{ \pm}\right)$directly, as shown in Figure 11.

Thus, we seek the delta-shock solution of the form (2.7) for (1.3) and (1.4). Without loss of generality, it suffices to solve the generalized Rankine-Hugoniot relation (2.8) with the following initial data

$$
x=0: y(0)=0, \quad u_{\delta}(0)=u_{0}, \quad v_{\delta}(0)=v_{0}, \quad w(0)=w_{0}
$$

satisfying $v_{+} / u_{+}<v_{0} / u_{0}<v_{-} / u_{-}$.

By a routine but rather tedious calculation as done in Subcase 3.2.1, one can solve from the generalized Rankine-Hugoniot relation (2.8) and (3.36) that

$$
\left\{\begin{array}{l}
y(x)=\frac{\left[\frac{n u v}{1-u^{2} / c^{2}-v^{2} / c^{2}}\right] x+\frac{w_{0} u_{0}^{2}}{1-u_{0}^{2} / c^{2}-v_{0}^{2} / c^{2}}-\sqrt{Q}}{\left[\frac{n u^{2}}{1-u^{2} / c^{2}-v^{2} / c^{2}}\right]}, \\
w(x)=\frac{1-u_{\delta}^{2}(x) / c^{2}-v_{\delta}^{2}(x) / c^{2}}{u_{\delta}^{2}(x)} \sqrt{Q}=\frac{\sqrt{Q}}{M^{2}(x)} \\
u_{\delta}(x)=\frac{M(x)}{\sqrt{1+M^{2}(x) / c^{2}+N^{2}(x) / c^{2}}} \\
v_{\delta}(x)=\frac{N(x)}{\sqrt{1+M^{2}(x)^{2} / c^{2}+N^{2}(x) / c^{2}}}
\end{array}\right.
$$

when $\left[\frac{n u^{2}}{1-u^{2} / c^{2}-v^{2} / c^{2}}\right] \neq 0$, in which

$$
Q=\frac{n_{-} n_{+}\left(u_{-} v_{+}-u_{+} v_{-}\right)^{2}}{\left(1-u_{-}^{2} / c^{2}-v_{-}^{2} / c^{2}\right)\left(1-u_{+}^{2} / c^{2}-v_{+}^{2} / c^{2}\right)} x^{2}+\frac{w_{0}^{2} u_{0}^{4}}{\left(1-u_{0}^{2} / c^{2}-v_{0}^{2} / c^{2}\right)^{2}}
$$




$$
+\frac{2 w_{0} u_{0}}{1-u_{0}^{2} / c^{2}-v_{0}^{2} / c^{2}}\left(\left[\frac{n u v}{1-u^{2} / c^{2}-v^{2} / c^{2}}\right] u_{0}-\left[\frac{n u^{2}}{1-u^{2} / c^{2}-v^{2} / c^{2}}\right] v_{0}\right) x
$$

and

$$
M(x)=\frac{M_{1}(x)}{M_{2}(x)}, \quad N(x)=\frac{N_{1}(x)}{N_{2}(x)},
$$

with

$$
\left\{\begin{aligned}
M_{1}(x)= & {\left[\frac{n u^{2}}{1-u^{2} / c^{2}-v^{2} / c^{2}}\right] \sqrt{Q} } \\
M_{2}(x)= & \frac{n}{\sqrt{1-u_{-} / c^{2}-n_{+}^{2} / u_{+} v_{-}} \sqrt{1-u_{+}^{2} / v^{2}-v_{+}^{2} / c^{2}}}\left(\frac{u_{-}}{\sqrt{1-u_{-} / c^{2}-v_{-}^{2} / c^{2}}}-\frac{u_{+}}{\sqrt{1-u_{+}^{2} / c^{2}-v_{+}^{2} / c^{2}}}\right) x \\
& -\frac{w_{0} u_{0}}{\sqrt{1-u_{0}^{2} / c^{2}-v_{0}^{2} / c^{2}}}\left(\frac{u_{0}}{\sqrt{1-u_{0}^{2} / c^{2}-v_{0}^{2} / c^{2}}}\left[\frac{n u}{\sqrt{1-u^{2} / c^{2}-v^{2} / c^{2}}}\right]-\left[\frac{n u^{2}}{1-u^{2} / c^{2}-v^{2} / c^{2}}\right]\right) \\
& +\left[\frac{n u}{\sqrt{1-u^{2} / c^{2}-v^{2} / c^{2}}}\right] \sqrt{Q} .
\end{aligned}\right.
$$

and

$$
\left\{\begin{aligned}
N_{1}(x)= & \frac{n_{-} n_{+}\left(u_{+} v_{-}-u_{-} v_{+}\right)^{2}}{\left(1-u_{-} / c^{2}-v_{-}^{2} / c^{2}\right)\left(1-u_{+}^{2} / c^{2}-v_{+}^{2} / c^{2}\right)} x-\left[\frac{n u v}{1-u^{2} / c^{2}-v^{2} / c^{2}}\right] \sqrt{Q} \\
& +\frac{w_{0} u_{0}}{1-u_{0}^{2} / c^{2}-v_{0}^{2} / c^{2}}\left(u_{0}\left[\frac{n u v}{1-u^{2} / c^{2}-v^{2} / c^{2}}\right]-v_{0}\left[\frac{n u^{2}}{1-u^{2} / c^{2}-v^{2} / c^{2}}\right]\right), \\
N_{2}(x)= & \frac{n_{-} n_{+}\left(u_{-} v_{+}-u_{+} v_{-}\right)}{\sqrt{1-u_{-} / c^{2}-v_{-}^{2} / c^{2}} \sqrt{1-u_{+}^{2} / c^{2}-v_{+}^{2} / c^{2}}}\left(\frac{u_{-}}{\sqrt{1-u_{-} / c^{2}-v_{-}^{2} / c^{2}}}-\frac{u_{+}}{\sqrt{1-u_{+}^{2} / c^{2}-v_{+}^{2} / c^{2}}}\right) x \\
& +\frac{w_{0} u_{0}}{\sqrt{1-u_{0}^{2} / c^{2}-v_{0}^{2} / c^{2}}}\left(\frac{u_{0}}{\sqrt{1-u_{0}^{2} / c^{2}-v_{0}^{2} / c^{2}}}\left[\frac{n u}{\sqrt{1-u^{2} / c^{2}-v^{2} / c^{2}}}\right]-\left[\frac{n u^{2}}{1-u^{2} / c^{2}-v^{2} / c^{2}}\right]\right) \\
& -\left[\frac{n u}{\sqrt{1-u^{2} / c^{2}-v^{2} / c^{2}}}\right] \sqrt{Q} .
\end{aligned}\right.
$$

The solutions for $\left[\frac{n u^{2}}{1-u^{2} / c^{2}-v^{2} / c^{2}}\right]=0$ can be similarly obtained, so we omit it here.

Remark 3.1. It is observed that, if $w_{0}=0, u_{0}=0$ and $v_{0}=0$, the structure of the delta-shock solution $\left(y(x), w(x), u_{\delta}(x), v_{\delta}(x)\right)$ obtained in (3.37)-(3.41) is in accordance with that in (2.12)-(2.13). This implies that the solutions constructed here are stable under some viscous perturbations.

So far, we have discussed all kinds of interactions of waves and established the global structures of solutions to the perturbed Riemann problem (1.3) and (1.5). Then, via taking $\epsilon \rightarrow 0^{+}$in the solutions of (1.3) and (1.5), we obtain the solutions of the Riemann problem (1.3) with delta initial data (1.4) technically. Our results can be summarized as follows.

Theorem 3.2. The solutions of (1.3) and (1.4) can be obtained by letting $\epsilon \rightarrow 0^{+}$in the solutions of (1.3) and (1.5). Moreover, the solutions of (1.3) and (1.4) are exactly corresponding to those of the Riemann problem (1.3) and $(2.1)$ when $\left(w_{0}, u_{0}, v_{0}\right)=(0,0,0)$, which implies that the solutions constructed here are stable under some perturbations. 


\section{REFERENCES}

[1] G. Chen, Overtaking of shocks of the same kind in the isentropic steady supersonic plane flow. Acta Math. Sci. 7 (1987) 311-327.

[2] J. Chen, G. Lai and J. Zhang, Boundary value problems for the 2D steady relativistic Euler equations with general equation of state. Nonlinear Anal.: Theory, Methods Appl. 175 (2018) 56-72.

[3] H. Cheng and H. Yang, Delta shock waves as limits of vanishing viscosity for 2-D steady pressureless isentropic flow. Acta Appl. Math. 113 (2011) 323-348.

[4] E. Weinan, Yu.G. Rykov and Ya.G. Sinai, Generalized variational principles, global weak solutions and behavior with random initial data for systems of conservation laws arising in adhesion particle dynamics. Commun. Math. Phys. 177 (1996) 349-380.

[5] L. Guo, T. Li, L. Pan and X. Han, The Riemann problem with delta initial data for the one-dimensional Chaplygin gas equations with a source term. Nonlinear Anal.: Real World Appl. 41 (2018) 588-606.

[6] L. Guo, L. Pan and G. Yin, The perturbed Riemann problem and delta contact discontinuity in chromatography equations. Nonlinear Anal.: Theory, Methods Appl. 106 (2014) 110-123.

[7] L. Guo and G. Yin, The Riemann problem with Delta initial data for the one-dimensional transport equations. B. Malays. Math. Sci. Soc. 38 (2015) 219-230.

[8] G. Lai and C. Shen, Characteristic decompositions and boundary value problems for two-dimensional steady relativistic Euler equations. Math. Methods Appl. Sci. 37 (2014) 136-147.

[9] J. Li and Z. Shao, The Riemann problem with delta initial data for the zero-pressure relativistic Euler equations. Acta Math. Sci. 34 (2014) 1083-1092.

[10] J. Li, S. Yang and T. Zhang, The two-dimensional Riemann problem in gas dynamics. In Vol. 98 of Pitman Monographs and Surveys in Pure and Applied Mathematics. Longman Scientific and Technical (1998).

[11] L. Luan, J. Chen and J. Liu, Two dimensional relativistic Euler equations in a convex duct. J. Math. Anal. Appl. 461 (2018) 1084-1099.

[12] M. Nedeljkov and M. Oberguggenberger, Interactions of delta shock waves in a strictly hyperbolic system of conservation laws. J. Math. Anal. Appl. 344 (2008) 1143-1157.

[13] S.F. Shandarin and Y.B. Zeldovich, The large-scale structure of the universe: turbulence, intermittence, structures in selfgravitating medium. Rev. Mod. Phys. 61 (1989) 185-220.

[14] Z. Shao, Riemann problem with delta initial data for the isentropic relativistic Chaplygin Euler equations. Z. Angew. Math. Phys. 67 (2016) 66.

[15] Z. Shao, The Riemann problem for the relativistic full Euler system with generalized Chaplygin proper energy density-pressure relation. Z. Angew. Math. Phys. 69 (2018) 44.

[16] C. Shen, The Riemann problem for the pressureless Euler system with the Coulomb-like friction term. IMA J. Appl. Math. 81 (2016) 76-99.

[17] M. Sun, Interactions of delta shock waves for the chromatography equations. Appl. Math. Lett. 26 (2013) 631-637.

[18] L. Wang, The Riemann problem with Delta data for zero-pressure gas dynamics. Chin. Ann. Math. 37B (2016) 441-450.

[19] Z. Wang and Q. Zhang, The Riemann problem with delta initial data for the one-dimensional Chaplygin gas equations. Acta Math. Sci. 3 (2012) 825-841.

[20] H. Yang, Riemann problems for a class of coupled hyperbolic systems of conservation laws. J. Differ. Equ. 159 (1999) $447-484$.

[21] H. Yang, Generalized plane delta-shock waves for $n$-dimensional zero-pressure gas dynamics. J. Math. Anal. Appl. 260 (2001) $18-35$.

[22] H. Yang and W. Sun, The Riemann problem with delta initial data for a class of coupled hyperbolic systems of conservation laws. Nonlinear Anal.: Theory, Methods Appl. 67 (2007) 3041-3049.

[23] Q. Zhang, Interactions of delta shock waves and stability of Riemann solutions for nonlinear chromatography equations. Z. Angew. Math. Phys. 67 (2016) 15.

[24] Y. Zhang and Y. Zhang, Riemann problem for a two-dimensional steady pressureless relativistic Euler equations. Math. Nachr. in press.

[25] Y. Zhang and Y. Zhang, Generalized plane delta shock waves for the $n$-dimensional zero-pressure gas dynamics with energy conservation law. Indian J. Pure Appl. Math. 50 (2019) 1067-1086.

[26] Y. Zhang and Y. Zhang, The Riemann problem with delta initial data for the two-dimensional steady zero-pressure adiabatic flow. Rocky Mountain J. Math. 49 (2019) 2395-2418. 